\title{
Deterministic and Stochastic Dynamics of COVID-19: The Case Study of Italy and Spain
}

\author{
Akhil Kumar Srivastav $\mathbb{D}^{1},{ }^{1}$ Nico Stollenwerk $\mathbb{D}^{1},{ }^{1,2}$ and Maíra Aguiar $\mathbb{D}^{1,2,3}$ \\ ${ }^{1}$ Basque Center for Applied Mathematics, BCAM, Bilbao, Spain \\ ${ }^{2}$ Dipartimento di Matematica, Universita degli Studi di Trento, Italy \\ ${ }^{3}$ Ikerbasque, Basque Foundation for Science, Bilbao, Spain \\ Correspondence should be addressed to Akhil Kumar Srivastav; asrivastav@bcamath.org
}

Received 15 September 2021; Accepted 5 December 2021; Published 13 February 2022

Academic Editor: Kuo Shou Chiu

Copyright (c) 2022 Akhil Kumar Srivastav et al. This is an open access article distributed under the Creative Commons Attribution License, which permits unrestricted use, distribution, and reproduction in any medium, provided the original work is properly cited.

\begin{abstract}
In December 2019, a severe respiratory syndrome (COVID-19) caused by a new coronavirus (SARS-CoV-2) was identified in China and spread rapidly around the globe. COVID-19 was declared a pandemic by the World Health Organization (WHO) in March 2020. With eventually substantial global underestimation, more than 225 million cases were confirmed by the end of August 2021, counting more than 4.5 million deaths. COVID-19 symptoms range from mild (or no symptoms) to severe illness, with disease severity and death occurring according to a hierarchy of risks, with age and preexisting health conditions enhancing the risks of disease severity manifestation. In this paper, a mathematical model for COVID-19 transmission is proposed and analyzed. The model stratifies the studied population into two groups, older and younger. Applied to the COVID-19 outbreaks in Spain and in Italy, we find the disease-free equilibrium and the basic reproduction number for each case study. A sensitivity analysis to identify the key parameters which influence the basic reproduction number, and hence regulate the transmission dynamics of COVID-19, is also performed. Finally, the model is extended to its stochastic counterpart to encapsulate the variation or uncertainty found in the transmissibility of the disease. We observe the variability of the infectious population finding its distribution at a given time, demonstrating that for small populations, stochasticity will play an important role.
\end{abstract}

\section{Introduction}

The coronavirus pandemic and its emerging variants are currently a major global public health threat. Globalisation has speeded up the spread of infections over a short period of time. This has an impact on the public healthcare system and is also detrimental to the economic development of many countries. Since the outbreaks began, more than 215 million cases were confirmed by mid of August 2021, with more than 4 million deaths [1].

COVID-19 symptoms can range from mild (or no symptoms) to severe illness, with disease severity and death occurring according to a hierarchy of risks, with age and preexisting health conditions enhancing risks of disease severity [2]. Vaccines against COVID-19 have been developed in record time and are now globally distributed [3-8]. The analysis of the impact of different vaccine administration is ongoing, mostly using previous research experiences applied to other infectious diseases [9-17] and will be discussed in detail in our forthcoming publications $[18,19]$.

As an example of the impact of the pandemic in Europe, Spain has reported, up to date, more than 4.7 million cases with around 83 thousand deaths [20], while in Italy, although the total number of cases are similar, 4.5 million, a higher mortality rate was reported, counting more than 128 thousand deaths [21]. It is important to notice that mortality is higher in older than younger individuals in all populations [22, 23].

As the COVID-19 pandemic progressed, research on mathematical modeling became imperative and very influential to understand the epidemiological dynamics of disease spreading. Task forces were created to assist public health 
managers and governments, with many research papers being recently published. Applied to the outbreaks in the Basque Country, Spain, a flexible framework was developed within the so-called COVID-19 Basque Modeling Task Force (BMTF). As an extension of the basic SHAR (Susceptible-Hospitalized-Asymptomatic-Recovered) model, the SHARUCD models were parameterized and validated with epidemiological data continuously collected and provided by the Basque Health Department and the Basque Health Service (Osakidetza) and have been used (up until now) to monitor COVID-19 spreading and control over the course of the pandemic [18, 19, 24-28]. Modeling refinements and results on the evolution of the epidemic in the Basque Country are regularly updated and publicly available on the "SHARUCD Dashboard" [29].

Over the course of the pandemic, a broad spectrum of research has been produced, e.g., statistical work using two common approaches, the SIR model and a log-linear model, analyzing the available empirical data and estimating the reproduction values for Spain and Italy countries [30]. Deterministic and stochastic models were developed [31, 32] to study the effects of facial masks and hospitalization of symptomatic people and asymptomatic quarantine of people on the prevalence of the coronavirus outbreak in India [31] and the transmission dynamics of the COVID19 in Wuhan, China [32]. And still, many questions remain to be investigated.

In this paper, we present a mathematical model to describe COVID-19 dynamics in Spain and Italy. The population is divided into two groups, young and old. Considering simple mass action type incidence, the model is formulated by assuming that the course COVID-19 infection leads to a different outcome for the elderly population as compared to the younger population. This paper is organized as follows. Section 2 describes the model formulation, followed by the model analysis in Section 3, showing the existence of equilibrium and basic reproduction number. Section 4 presents the results for the sensitivity analysis for the parameters involved in reproduction number. Section 5 describes impact of different parameter on COVID-19 prevalence. Section 6 presents the stochastic modeling approach, and in Section 7, the simulation results are shown. Finally, in Section 8 , we conclude this work, with a discussion on both modeling approaches.

\section{The Model}

This model is a refinement of the model proposed by Srivastav et al. [33]. A new parameter $\varepsilon$ is introduced to differentiate the infectivity of young infected individuals $\left(I_{1}\right)$ with respect to the baseline infectivity of elderly individuals $I_{2}(t$ ) in a population of $N$ individuals.

The value of $\varepsilon$ can be tuned to reflect different situations: a value of $\varepsilon>1$ reflects the fact that young individuals, which are likely to develop mild disease and higher mobility, have larger infectivity, than elderly individuals, which are at higher risk of developing severe disease and are more likely to be detected and isolated [24]. On the other hand, $\varepsilon<1$ indicates that young individuals have smaller infectivity than elderly individuals, and that could be justified due to lower or higher viral load during the infection, which is correlated with disease symptoms. Here, the assumption relies on the epidemiological observation of young population developing mild or no symptoms with lower viral load, affecting disease transmissibility, versus severe disease and higher viral load, mostly observed in older ages.

The total human population $N(t)$ is divided into eight compartments, stratified into two age classes, namely, young and old: susceptible $S_{1}(t)$ and $S_{2}(t)$, exposed $E_{1}(t)$ and $E_{2}(t)$, and infected $I_{1}(t)$ and $I_{2}(t)$ for the young and for the old, respectively. Two extra classes to accommodate individuals from both age groups are also considered: quarantined/hospitalized $H(t)$ for those identified as COVID-19-positive case with symptoms and needing medical assistance and finally the recovered individual class $R(t)$.

We assume that the transitions/movements, from one disease related class to another, are different between the elderly and the young individuals. However, the $H(t)$ class includes both groups. For the mathematical modeling framework development, we make the following assumptions:

(1) Total population $N$ is constant

(2) The susceptible young individuals $S_{1}$ become exposed to the infection and join the young exposed class $E_{1}$ on effective contacts with infectious human population $I_{1}, I_{2}$ and $H$ at rates $\beta_{1}$ and $\beta_{3}$, respectively, with $\beta_{3}<\beta_{1}$

(3) Exposed people $E_{1}$ will move to $I_{1}$ with rate of $\eta_{1}$. If young exposed people $E_{1}$ will get contact with $I_{1}, I_{2}$ unknowingly, then exposed people $E_{1}$ will move fast into $I_{1}$ class with rate $\gamma_{1}$

(4) The susceptible old individuals $S_{2}$ become exposed to the infection and join the old exposed class $E_{2}$ on effective contacts with infectious human population $I_{1}, I_{2}$ and $(H)$ at the rates $\beta_{2}$ and $\beta_{4}$, respectively, with $\beta_{4}<\beta_{2}$

(5) Exposed people $E_{2}$ will move to $I_{2}$ with rate of $\eta_{2}$; if old exposed people $E_{2}$ will get contact with $I_{1}, I_{2}$ unknowingly, then exposed people $E_{2}$ will move fast in $I_{2}$ with rate $\gamma_{2}$

(6) Infected, young $I_{1}$ and old $I_{2}$, will move to hospitalized class $(H)$ with rates $v_{1}$ and $v_{2}$, respectively

(7) Hospitalized people $(H)$ will get recovered from COVID-19 and join recovered class $(R)$ with rate $\alpha$

The schematic diagram of our proposed model is shown in Figure 1, and the mathematical model is given as follows:

$$
\frac{d S_{1}}{d t}=-\beta_{1} S_{1}\left(\varepsilon I_{1}+I_{2}\right)-\beta_{3} S_{1} H
$$

$$
\frac{d E_{1}}{d t}=\beta_{1} S_{1}\left(\varepsilon I_{1}+I_{2}\right)+\beta_{3} S_{1} H-\gamma_{1} E_{1}\left(\varepsilon I_{1}+I_{2}\right)-\eta_{1} E_{1}
$$




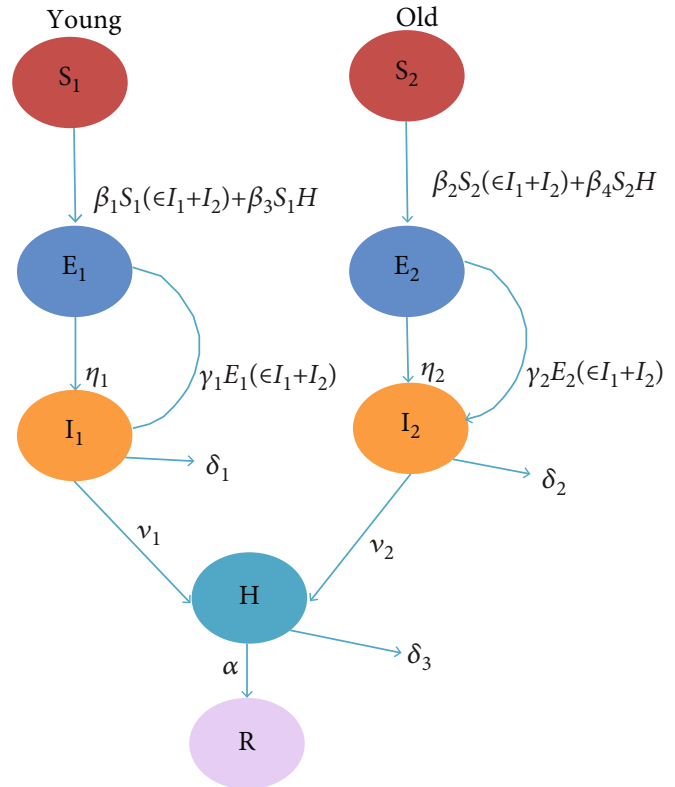

Figure 1: Schematic diagram of the model.

$$
\begin{gathered}
\frac{d I_{1}}{d t}=\eta_{1} E_{1}+\gamma_{1} E_{1}\left(\varepsilon I_{1}+I_{2}\right)-v_{1} I_{1}-\delta_{1} I_{1}, \\
\frac{d S_{2}}{d t}=-\beta_{2} S_{2}\left(\varepsilon I_{1}+I_{2}\right)-\beta_{4} S_{2} H
\end{gathered}
$$

$$
\begin{gathered}
\frac{d E_{2}}{d t}=\beta_{2} S_{2}\left(\varepsilon I_{1}+I_{2}\right)+\beta_{4} S_{2} H-\gamma_{2} E_{2}\left(\varepsilon I_{1}+I_{2}\right)-\eta_{2} E_{2} \\
\frac{d I_{2}}{d t}=\eta_{2} E_{2}+\gamma_{2} E_{2}\left(\varepsilon I_{1}+I_{2}\right)-\delta_{2} I_{2}-v_{2} I_{2} \\
\frac{d H}{d t}=v_{1} I_{1}+v_{2} I_{2}-\delta_{3} H-\alpha H \\
\frac{d R}{d t}=\alpha H .
\end{gathered}
$$

Here, all the parameters are positive and the description of these parameters is given in Table 1. Parameter values given in Tables 2 and 3 are already defined in [33].

\section{Analysis of the Model}

We consider the system (1) and find the disease-free equilibrium. For our model, we have disease-free equilibrium as

$$
E_{0}=\left(S_{1}{ }^{0}, E_{1}{ }^{0}, I_{1}{ }^{0}, S_{2}{ }^{0}, E_{2}{ }^{0}, I_{1}{ }^{0}, H^{0}, R^{0}\right)=\left(N_{1}^{0}, 0,0, N_{2}^{0}, 0,0,0,0\right) .
$$

We find the basic reproduction number $R_{0}$ by following the next-generation matrix method described in [38]. Following the same notations as in [38], we find the vector $\mathscr{F}$
TABLE 1: Description of parameters.

\begin{tabular}{lc}
\hline Parameter & Description \\
\hline$\beta_{1}$ & Transmission rate from $I_{1}$ or $I_{2}$ to $S_{1}$ \\
$\beta_{2}$ & Transmission rate from $I_{1}$ or $I_{2}$ to $S_{2}$ \\
$\beta_{3}$ & Transmission rate from $H$ to $S_{1}$ \\
$\beta_{4}$ & Transmission rate from $H$ to $S_{2}$ \\
$\varepsilon$ & Modification parameter \\
$\delta_{1}$ & Disease-related death rate in $I_{1}$ compartment \\
$\delta_{2}$ & Disease-related death rate in $I_{2}$ compartment \\
$\delta_{3}$ & Disease-related death rate in $H$ compartment \\
$v_{1}$ & Rate of detection/quarantine in $I_{1}$ compartment \\
$v_{2}$ & Rate of detection/quarantine in $I_{2}$ compartment \\
$\eta_{1}$ & Rate of progression of individuals from $E_{1}$ to $I_{1}$ \\
$\eta_{2}$ & Rate of progression of individuals from $E_{1}$ to $I_{1}$ \\
$\gamma_{1}$ & Rate of reinfection in $E_{1}$ compartment \\
$\gamma_{2}$ & Rate of reinfection in $E_{2}$ compartment \\
$\alpha$ & Recovery rate of quarantine/hospitalized people \\
\hline
\end{tabular}

and $\mathscr{V}$ as follows:

$$
\begin{gathered}
\mathscr{F}=\left(\begin{array}{c}
\beta_{1} S_{1}\left(\varepsilon I_{1}+I_{2}\right)+\beta_{3} S_{1} H \\
\beta_{2} S_{2}\left(\varepsilon I_{1}+I_{2}\right)+\beta_{4} S_{2} H \\
0 \\
0 \\
0
\end{array}\right), \\
\mathscr{V}=\left(\begin{array}{c} 
\\
\gamma_{1} E_{1}\left(\varepsilon I_{1}+I_{2}\right)+\eta_{1} E_{1} \\
\gamma_{2} E_{2}\left(\varepsilon I_{1}+I_{2}\right)+\eta_{2} E_{2} \\
-\eta_{1} E_{1}-\gamma_{1} E_{1}\left(\varepsilon I_{1}+I_{2}\right)+v_{1} I_{1}+\delta_{1} I_{1} \\
-\gamma_{2} E_{2}\left(\varepsilon I_{1}+I_{2}\right)+\delta_{2} I_{2}+v_{2} I_{2} \\
-v_{1} I_{1}-v_{2} I_{2}+\left(\delta_{3}+\alpha\right) Q
\end{array}\right) .
\end{gathered}
$$

$$
F=\text { Jacobian of } \mathscr{F} \text { at }
$$

$$
E_{0}=\left(\begin{array}{ccccc}
0 & 0 & \beta_{1} \varepsilon S_{1}^{0} & \beta_{1} S_{1}^{0} & \beta_{3} S_{1}^{0} \\
0 & 0 & \beta_{2} \varepsilon S_{2}^{0} & \beta_{2} S_{2}^{0} & \beta_{4} S_{2}^{0} \\
0 & 0 & 0 & 0 & 0 \\
0 & 0 & 0 & 0 & 0 \\
0 & 0 & 0 & 0 & 0
\end{array}\right),
$$


TABLE 2: Values of parameters.

\begin{tabular}{lc}
\hline Parameter & Value \\
\hline$\beta_{3}$ & 0.000513 assumed \\
$\beta_{4}$ & 0.000672 assumed \\
$\gamma_{1}$ & 0.14 assumed \\
$\eta_{1}$ & $0.08(1-14$ days $)[34]$ \\
$\eta_{2}$ & $0.1(1-14$ days $)[34]$ \\
$\gamma_{2}$ & 0.2 assumed \\
$\delta_{1}$ & 0.013 assumed \\
$\delta_{2}$ & 0.014 assumed \\
$\delta_{3}$ & $0.0150 .001-0.1[35]$ \\
$\alpha$ & $0.071(14-28$ days $)[36,37]$ \\
\hline
\end{tabular}

TABLE 3: Values of parameters.

\begin{tabular}{lcc}
\hline Country & Estimated values & Value of $R_{0}$ \\
\hline & $\beta_{1}=0.0028$ & \\
Italy & $\beta_{2}=0.0086$ & 2.644 \\
& $v_{1}=0.031$ & \\
& $v_{2}=0.058$ & \\
& $\beta_{1}=0.0024$ & \\
& $\beta_{2}=0.0085$ & 2.137 \\
Spain & $v_{1}=0.043$ & \\
& $v_{2}=0.053$ &
\end{tabular}

and $V=$ Jacobian of $\mathscr{V}$ at

$$
E_{0}=\left(\begin{array}{ccccc}
\eta_{1} & 0 & 0 & 0 & 0 \\
0 & \eta_{2} & 0 & 0 & 0 \\
-\eta_{1} & 0 & \left(v_{1}+\delta_{1}\right) & 0 & 0 \\
0 & -\eta_{2} & 0 & \left(\nu_{2}+\delta_{2}\right) & 0 \\
0 & 0 & -v_{1} & -v_{2} & \left(\delta_{3}+\alpha\right)
\end{array}\right) .
$$

And it follows that

$$
F V^{-1}=\left(\begin{array}{ccccc}
a_{11} & a_{12} & a_{13} & a_{14} & a_{15} \\
a_{21} & a_{22} & a_{23} & a_{24} & a_{25} \\
0 & 0 & 0 & 0 & 0 \\
0 & 0 & 0 & 0 & 0 \\
0 & 0 & 0 & 0 & 0
\end{array}\right)
$$

where

$$
\begin{aligned}
& a_{11}=\frac{\beta_{1} \varepsilon S_{1}^{0}}{v_{1}+\delta_{1}}+\frac{\beta_{3} v_{1} S_{1}^{0}}{\left(\delta_{3}+\alpha\right)\left(v_{1}+\delta_{1}\right)}, \\
& a_{12}=\frac{\beta_{1} S_{1}^{0}}{v_{2}+\delta_{2}}+\frac{\beta_{3} v_{2} S_{1}^{0}}{\left(\delta_{3}+\alpha\right)\left(v_{2}+\delta_{2}\right)}, \\
& a_{13}=\frac{\beta_{1} \varepsilon S_{1}^{0}}{v_{1}+\delta_{1}}+\frac{\beta_{3} v_{1} S_{1}^{0}}{\left(\delta_{3}+\alpha\right)\left(v_{1}+\delta_{1}\right)}, \\
& a_{14}=\frac{\beta_{1} S_{1}^{0}}{v_{2}+\delta_{2}}+\frac{\beta_{3} v_{2} S_{1}^{0}}{\left(\delta_{3}+\alpha\right)\left(v_{2}+\delta_{2}\right)}, \\
& a_{21}=\frac{\beta_{2} \varepsilon S_{2}^{0}}{v_{1}+\delta_{1}}+\frac{\beta_{15} v_{1} S_{2}^{0}}{\alpha+\delta_{3}}, \\
& a_{22}=\frac{\beta_{2} S_{2}^{0}}{v_{2}+\delta_{2}}+\frac{\beta_{4} v_{2} S_{2}^{0}}{\left(\delta_{3}+\alpha\right)\left(v_{2}+\delta_{2}\right)}, \\
& a_{23}=\frac{\beta_{2} \varepsilon S_{2}^{0}}{v_{1}+\delta_{1}}+\frac{\beta_{4} v_{1} S_{2}^{0}}{\left(\delta_{3}+\alpha\right)\left(v_{1}+\delta_{1}\right)}, \\
& a_{24}=\frac{\beta_{2} S_{2}^{0}}{v_{2}+\delta_{2}}+\frac{\beta_{4} v_{2} S_{2}^{0}}{\left(\delta_{3}+\alpha\right)\left(v_{2}+\delta_{2}\right)}, \\
& \alpha+\delta_{3} \cdot
\end{aligned}
$$

Three eigenvalues of the above matrix are zero and the remaining two are the roots of the following quadratic equation:

$$
\begin{gathered}
\lambda^{2}-\left(a_{11}+a_{22}\right) \lambda+a_{11} a_{22}-a_{12} a_{21}=0 \\
a_{11} a_{22}-a_{12} a_{21}=\frac{S_{1}^{0} S_{2}^{0}}{\left(\delta_{3}+\alpha\right)\left(v_{1}+\delta_{1}\right)\left(v_{2}+\delta_{2}\right)}\left[\left(\varepsilon v_{2}-v_{1}\right)\left(\beta_{1} \beta_{4}-\beta_{2} \beta_{3}\right)\right] .
\end{gathered}
$$

So the basic reproduction number $\left(R_{0}\right)$ is the positive root of the above quadratic and is given by

$$
R_{0}=\frac{R_{01}+\sqrt{R_{01}^{2}+4 R_{02}^{2}}}{2},
$$

where

$$
\begin{aligned}
& R_{01}=\frac{\beta_{1} \varepsilon S_{1}^{0}}{v_{1}+\delta_{1}}+\frac{\beta_{3} v_{1} S_{1}^{0}}{\left(\delta_{3}+\alpha\right)\left(v_{1}+\delta_{1}\right)}+\frac{\beta_{2} S_{2}^{0}}{v_{2}+\delta_{2}}+\frac{\beta_{4} S_{2}^{0} v_{2}}{\left(\delta_{3}+\alpha\right)\left(v_{2}+\delta_{2}\right)}, \\
& R_{02}=\sqrt{\frac{S_{1}^{0} S_{2}^{0}}{\left(\delta_{3}+\alpha\right)\left(v_{1}+\delta_{1}\right)\left(\nu_{2}+\delta_{2}\right)}\left[\left(\varepsilon v_{2}-v_{1}\right)\left(\beta_{1} \beta_{4}-\beta_{2} \beta_{3}\right)\right]} \text {. }
\end{aligned}
$$




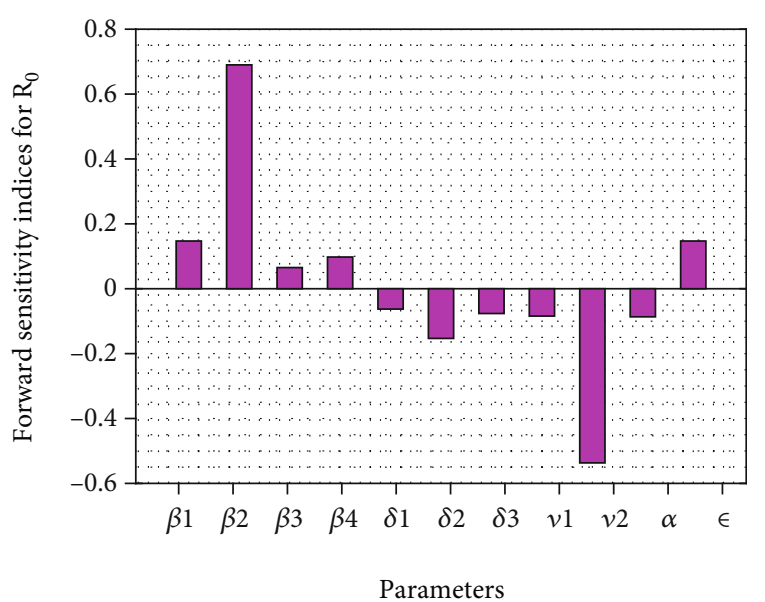

(a)

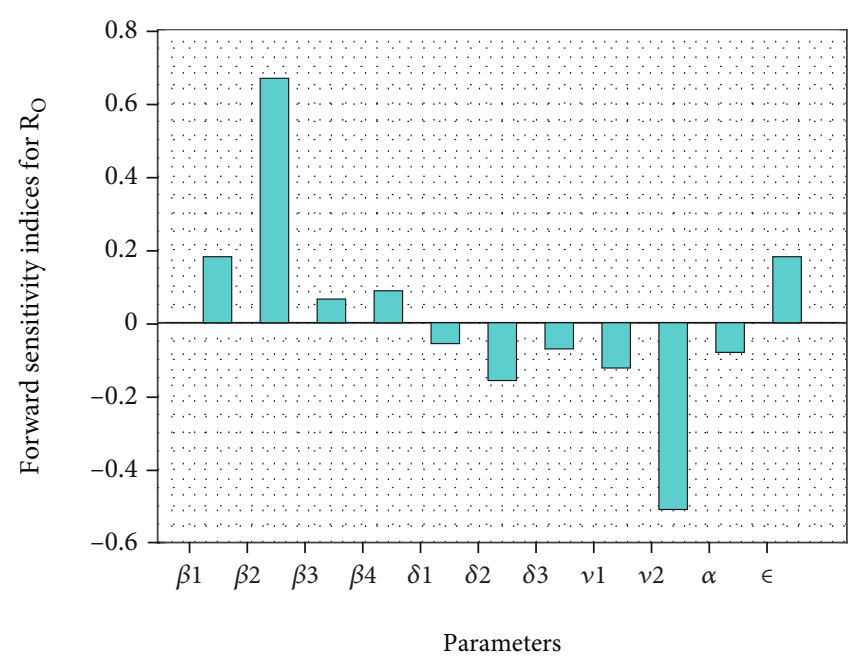

(b)

FIGURE 2: Forward sensitivity analysis of the parameters on $R_{0}$ in (a) Italy and in (b) Spain.
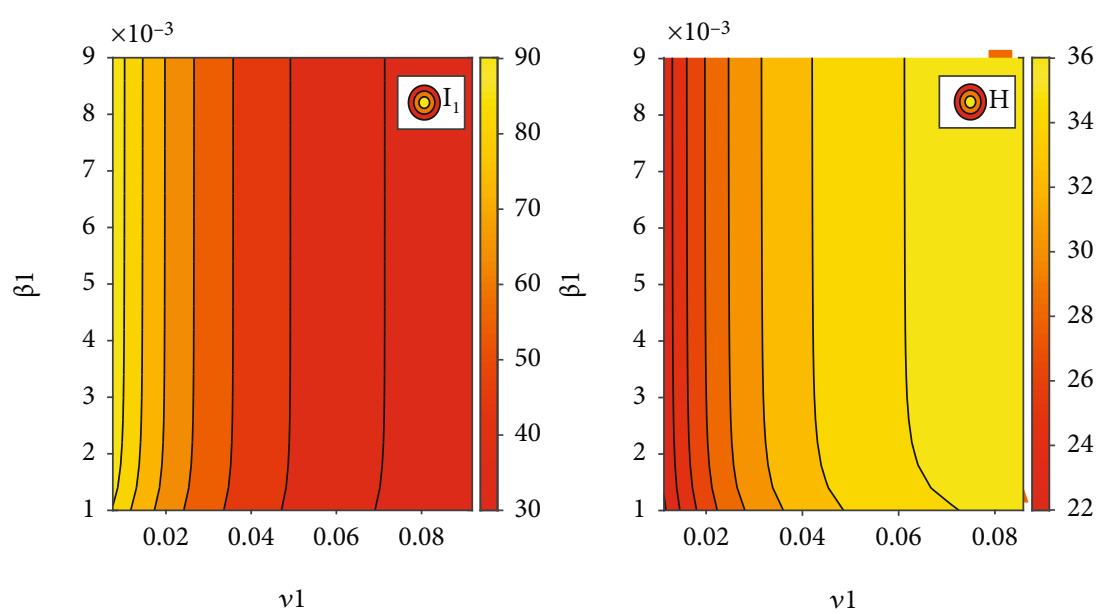

FIGURE 3: Contour plots representing the effects of $v_{1}$ and $\beta_{1}$ on $I_{1}$ (first column) and $H$ (second column) for Italy.

\section{Sensitivity Analysis}

We also perform a sensitivity analysis for the parameters involved in reproduction number $\left(R_{0}\right)$, which reflects that the increase or decrease in these parameter values will lead to the increase or decrease of $\left(R_{0}\right)$. The sensitivity of $R_{0}$ to different parameters is shown in Figure 2. This analysis is performed to evaluate which parameters have the highest impact on $R_{0}$ and hence being targeted as the most effective intervention measures for each case study. The sensitivity indices allow to measure the relative change in a variable when parameter changes. For that, we use the forward sensitivity index of a variable with respect to a given parameter, which is defined as the ratio of the relative change in the variable to the relative change in the parameter. If the variable is varying with respect to a parameter, then the sensitivity index is defined using partial derivatives [39]. The normalized forward sensitivity index of $R_{0}$, which is differentiable with respect to a given parameter $p$, is defined by

$$
\gamma_{p}^{R_{0}}=\frac{\partial R_{0}}{\partial p} \frac{p}{R_{0}} .
$$

The above formula can be used to compute the analytical expression for the sensitivity of $R_{0}$ to each parameter included in the system. From Figure 2, we can conclude that $\beta_{i}, v_{i}$ for $i=1,2$ and $p$, for both case study, Italy and Spain, are very sensitive parameters, with small changes in these parameters leading to a significant change in the value of $R_{0}$.

\section{Impact of Different Parameters on Prevalence of COVID-19}

From Figures 3 and 4, we observe that the increase of transmission rate $\beta_{1}$, from infected young and old individuals to 

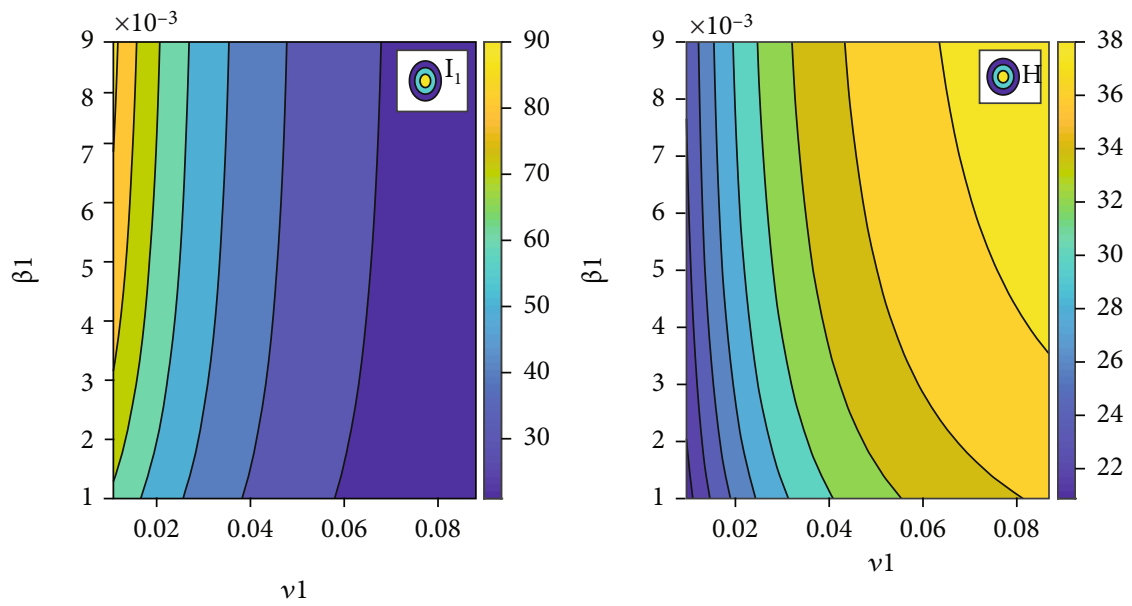

Figure 4: Contour plots representing the effects of $v_{1}$ and $\beta_{1}$ on $I_{1}$ (first column) and $H$ (second column) for Spain.
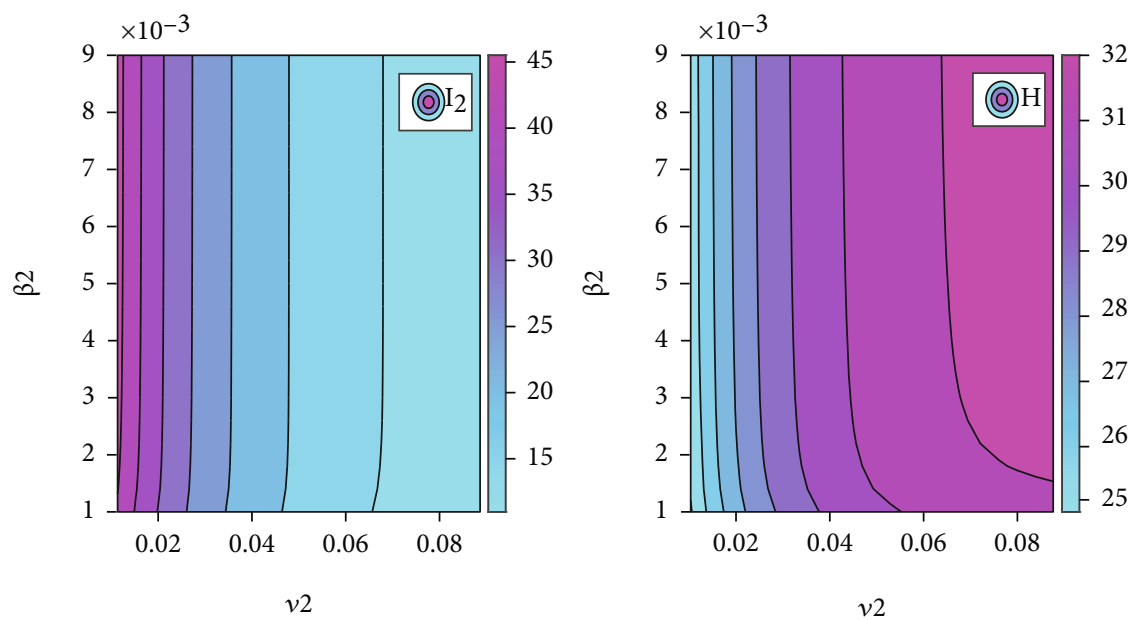

FIGURE 5: Contour plots representing the effects of $v_{1}$ and $\beta_{1}$ on $I_{1}$ (first column) and $H$ (second column) for Italy.
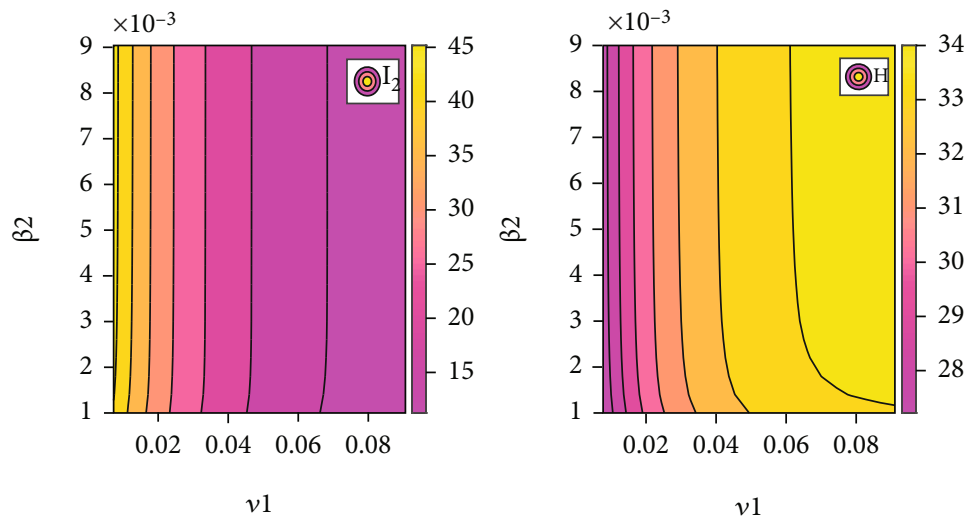

FIGURE 6: Contour plots representing the effects of $v_{1}$ and $\beta_{1}$ on $I_{1}$ (first column) and $H$ (second column) for Spain.

susceptible young individuals, leads to an increment of the infected young individuals in Italy and in Spain. However, the increase of $v_{1}$, the detection rate of infected young indi- viduals, will decrease the number of infections in the young human population class, but increasing the number of hospitalizations overall. Similarly as described above, Figures 5 


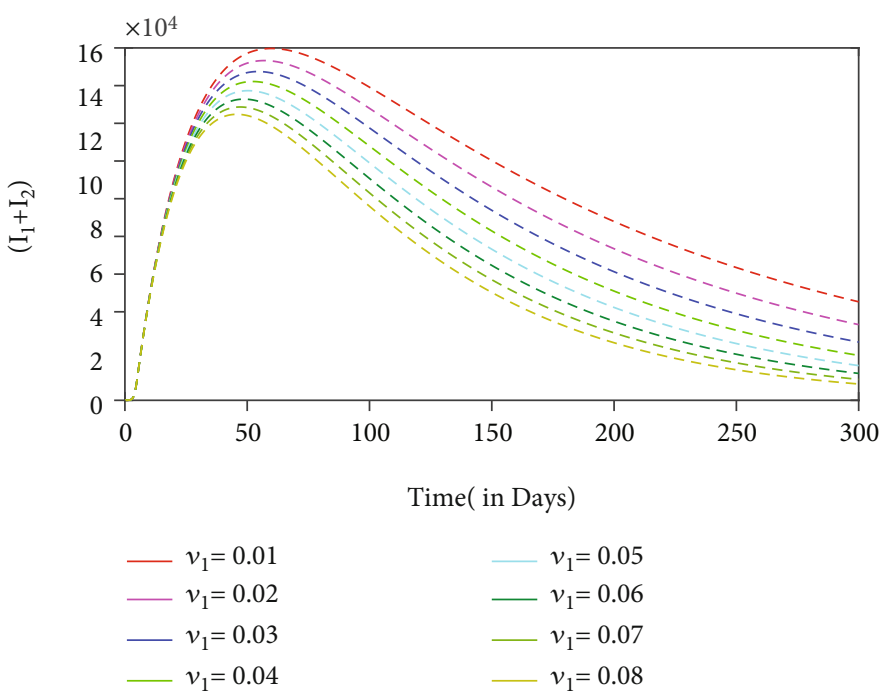

Figure 7: Variation of $\left(I_{1}+I_{2}\right)$ with time for different values of $v_{1}$.

and 6 show that the infections in old populations $I_{2}$ will increase as $\beta_{2}$, the transmission rate from infected young and old individuals to susceptible old individuals, increases. Nevertheless, the increase of $v_{2}$, the detection rate of infected old individuals, will decrease the number of infections in the old population, but also increasing the overall hospitalizations.

From these figures, we can conclude that controlling the transmission between human to human and increasing the detection rates, providing better treatment for the infected hospitalized people, will be of a major importance to control the epidemics in Italy and in Spain, with a significant decrease of number of infections in the population.

Finally, Figure 7 shows the effect of the variation of the parameter $v_{1}$ on the number of infections. As $v_{1}$ increases, the overall infected population decreases. Note that variations of the parameter $v_{2}$ show similar effects on the number of overall infections.

\section{Stochastic Model}

As all natural systems are prone to stochastic perturbations, we extended our deterministic model, equation system (1), to the corresponding stochastic model. The derivation of the stochastic model and its analysis are important when populations are small and hence with the dynamics being severely affected by small changes in the parameter values. Thus, for the initial phase of the disease outbreak, such as COVID-19, the stochastic model setup is the most appropriate modeling approach to be used for a local epidemiological evaluation.

The derivation of a stochastic differential equation (SDE) model is a diffusion approximation from the underlying state discrete Markov process [26, 40-44]. Let $X(t)=$ $\left(X_{1}(t), X_{2}(t), X_{3}(t), X_{4}(t), X_{5}(t), X_{6}(t), X_{7}(t), X_{8}(t)\right)^{T}$ be a continuous random for $\left(S_{1}(t), E_{1}(t), I_{1}(t), S_{2}(t), E_{2}(t), I_{2}(t), H(t), R(t)\right)^{T}$, where $T$ denotes the transpose of the matrix. Further, let $\Delta X=X(t+$
$\Delta t)-X(t)=\left(\Delta X_{1}, \Delta X_{2}, \Delta X_{3}, \Delta X_{4}, \Delta X_{5}(t), \Delta X_{6}(t), \Delta X_{7}(t)\right.$, $\left.\Delta X_{8}(t)\right)^{T}$ denote the random vector for the change in the random variables during time interval $\Delta t$. Here, we will write the transition maps which define all possible changes between states in the SDE model. Based on our deterministic model, see equation system (1), we see that there exist 19 possible changes between states in a small time interval $\Delta t$ (see Table 4). Here, it is emphasized that the one and only possible change is in the time $\Delta t$. For example, let us consider the case when one uninfected individual becomes infected by coronavirus. This will be given by the state change $\Delta X$, denoted by $\Delta X=(-1,1,0,0,0,0,0)$, and the change in its probability is given by

$$
\begin{aligned}
& \operatorname{prob}\left(\Delta X_{1}, \Delta X_{2}, \Delta X_{3}, \Delta X_{4}, \Delta X_{5}, \Delta X_{6}, \Delta X_{7}, \Delta X_{8}(t)\right) \\
& \quad=(-1,1,0,0,0,0,0,0) \mid\left(X_{1}, X_{2}, X_{3}, X_{4}, X_{5}, X_{6}, X_{7}, X_{8}\right) \\
& \quad=P_{1}=\beta X_{1} X_{2} \Delta t+O(t) .
\end{aligned}
$$

One can easily determine the expectation change $E(\Delta X)$ and its covariance matrix $V(\Delta X)$ associated with $\Delta X$ by neglecting the higher order terms $O(\Delta t)$. The expectation of $\Delta X$ is given by

$$
E(\Delta X)=\sum_{i=1}^{20} P_{i}(\Delta X)_{i} \Delta t=\left(\begin{array}{c}
-\beta_{1} X_{1}\left(\varepsilon X_{3}+X_{6}\right)-\beta_{3} X_{1} X_{7} \\
\beta_{1} X_{1}\left(\varepsilon X_{3}+X_{6}\right)+\beta_{3} X_{1} X_{7}-\gamma_{1} X_{1}\left(\varepsilon X_{3}+I_{6}\right)-\eta_{1} X_{2} \\
\eta_{1} X_{2}+\gamma_{1} X_{1}\left(\varepsilon X_{3}+I_{6}\right)-\left(v_{1}+\delta_{1}\right) X_{3} \\
-\beta_{2} X_{4}\left(\varepsilon X_{3}+X_{6}\right)-\beta_{4} X_{4} X_{7} \\
\beta_{2} X_{4}\left(\varepsilon X_{3}+X_{6}\right)+\beta_{4} X_{1} X_{7}-\gamma_{2} X_{5}\left(\varepsilon X_{3}+X_{6}\right)-\eta_{2} X_{5} \\
\eta_{2} X_{5}+\gamma_{2} X_{5}\left(\varepsilon X_{3}+X_{6}\right)-\left(v_{2}+\delta_{2}\right) X_{6} \\
v_{1} X_{3}+v_{2} X_{6}-\delta_{3} X_{7}-\alpha X_{7} \\
\alpha X_{7}
\end{array}\right) \Delta t
$$$$
=f\left(X_{1}, X_{2}, X_{3}, X_{4}, X_{5}, X_{6}, X_{7}, X_{8}\right) \Delta t \text {. }
$$ 
TABLe 4: Possible changes of states and their probabilities.

Possible state change

Probability of state change

$(\Delta X)_{1}=(-1,1,0,0,0,0,0,0)^{T}$

Change when people meet young infective $\left(I_{1}\right)$ and then population becomes exposed

$P_{1}=\beta_{1} \varepsilon X_{1} X_{3} \Delta t+O(\Delta t)$

$(\Delta X)_{2}=(-1,1,0,0,0,0,0,0)^{T}$

Change when people meet old infective $\left(I_{2}\right)$ and then population becomes exposed

$P_{2}=\beta_{1} X_{1} X_{6} \Delta t+O(\Delta t)$

$(\Delta X)_{2}=(-1,1,0,0,0,0,0,0,0)^{T}$

Change when people meet hospitalized $(H)$ and then population becomes exposed

$P_{3}=\beta_{3} X_{1} X_{7} \Delta t+O(\Delta t)$

$(\Delta X)_{4}=(0,-1,1,0,0,0,0,0)^{T}$

Change when young exposed with mild symptoms meet young infective $\left(I_{1}\right)$ and then population becomes

$P_{4}=\gamma_{1} \varepsilon X_{2} X_{3} \Delta t+O(\Delta t)$ infected

$(\Delta X)_{5}=(0,-1,1,0,0,0,0,0)^{T}$

Change when young exposed with mild symptoms meet old infective $\left(I_{2}\right)$ and then population becomes infected $(\Delta X)_{6}=(0,-1,1,0,0,0,0,0)^{T}$

Change when young exposed people move to young infected class $\left(I_{1}\right)$

$(\Delta X)_{7}=(0,0,-1,0,0,0,0,1,0)^{T}$

Change when young infective join hospitalized class

$(\Delta X)_{8}=(0,0,-1,0,0,0,0,0)^{T}$

Disease-related death rate of young infected population $\left(I_{1}\right)$

$(\Delta X)_{9}=(0,0,0,-1,1,0,0,0)^{T}$

Change when old people meet young infective $\left(I_{1}\right)$ and then population becomes exposed

$P_{5}=\gamma_{1} X_{2} X_{6} \Delta t+O(\Delta t)$

$(\Delta X)_{10}=(0,0,0,-1,1,0,0,0)^{T}$

Change when old people meet old infective $\left(I_{2}\right)$ and then population becomes exposed

$(\Delta X)_{11}=(0,0,0,-1,1,0,0,0)^{T}$

Change when old susceptible meet hospitalized $(H)$ and then population becomes exposed

$$
\begin{gathered}
P_{6}=\eta_{1} X_{2} \Delta t+O(\Delta t) \\
P_{7}=v_{1} X_{3} \Delta t+O(\Delta t) \\
P_{8}=\delta_{1} X_{3} \Delta t+O(\Delta t) \\
P_{9}=\beta_{2} \varepsilon X_{4} X_{3} \Delta t+O(\Delta t) \\
P_{10}=\beta_{2} X_{4} X_{6} \Delta t+O(\Delta t) \\
P_{11}=\beta_{4} X_{4} X_{7} \Delta t+O(\Delta t)
\end{gathered}
$$

$(\Delta X)_{12}=(0,0,0,0,-1,1,0,0)^{T}$

Change when old exposed with mild symptoms meet young infective $\left(I_{1}\right)$ and then population becomes infected

$(\Delta X)_{13}=(0,0,0,0,-1,1,0,0)^{T}$

Change when old exposed with mild symptoms meet old infective $\left(I_{2}\right)$ and then population becomes infected

$(\Delta X)_{14}=(0,0,0,0,-1,1,0,0)^{T}$

Change when young exposed people move to young infected class $\left(I_{1}\right)$

$P_{12}=\gamma_{2} \varepsilon X_{5} X_{3} \Delta t+O(\Delta t)$

$(\Delta X)_{15}=(0,0,0,0,0,-1,0,0)^{T}$

Disease-related death rate of young infected population $\left(I_{2}\right)$

$P_{13}=\gamma_{1} X_{5} X_{6} \Delta t+O(\Delta t)$

$(\Delta X)_{16}=(0,0,0,0,0,0,-1,1,0)^{T}$

Change when old infective join hospitalized class $(H)$

$$
\begin{aligned}
& P_{14}=\eta_{2} X_{2} \Delta t+O(\Delta t) \\
& P_{15}=\delta_{2} X_{3} \Delta t+O(\Delta t) \\
& P_{16}=v_{2} X_{6} \Delta t+O(\Delta t) \\
& P_{17}=\alpha X_{7} \Delta t+O(\Delta t) \\
& P_{18}=\delta_{3} X_{3} \Delta t+O(\Delta t) \\
& P_{19}=1-\sum_{i=1}^{18} \Delta t+O(\Delta t)
\end{aligned}
$$$$
\text { Change when hospitalized people }(H) \text { join recover class }
$$$$
(\Delta X)_{18}=(0,0,0,0,0,0,-1,0)^{T}
$$$$
\text { Disease-related death rate of hospitalized population }\left(I_{2}\right)
$$$$
(\Delta X)_{19}=(0,0,0,0,0,0,0,0,0,0)^{T}
$$ 
Note that the expectation vector and the function $f$ are in the same form as those in deterministic system (1). With the covariance matrix $V(\Delta X)=E\left((\Delta X)(\Delta X)^{T}\right)-E(\Delta X) E($ $\left.(\Delta X)^{T}\right)$ and $E(\Delta X) E\left((\Delta X)^{T}\right)=f(X)\left(f(X)^{T}\right)$, it can be approximated by $\Omega \times \Delta t$ by neglecting the term of $(\Delta t)^{2}$ such that

$$
\begin{aligned}
E\left((\Delta X)(\Delta X)^{T}\right) & =\sum_{i=1}^{19} P_{i}\left((\Delta X)_{i}(\Delta X)_{i}^{T}\right) \Delta t \\
& =\left(\begin{array}{cccccccc}
V_{11} & V_{12} & 0 & 0 & 0 & 0 & 0 & 0 \\
V_{21} & V_{22} & V_{23} & 0 & 0 & 0 & 0 & 0 \\
0 & V_{32} & V_{33} & 0 & 0 & 0 & V_{37} & 0 \\
0 & 0 & 0 & V_{44} & V_{45} & 0 & 0 & 0 \\
0 & 0 & 0 & V_{54} & V_{55} & V_{56} & 0 & 0 \\
0 & 0 & 0 & 0 & V_{65} & V_{66} & V_{67} & 0 \\
0 & 0 & V_{73} & 0 & 0 & V_{76} & V_{77} & V_{78} \\
0 & 0 & 0 & 0 & 0 & 0 & V_{87} & V_{88}
\end{array}\right) \cdot \Delta t \\
& =\Omega \cdot \Delta t,
\end{aligned}
$$

where

$$
\begin{gathered}
V_{11}=P_{1}+P_{2}+P_{3}=\beta_{1}\left(\varepsilon X_{1} X_{3}+X_{1} X_{6}\right)+\beta_{3} X_{1} X_{7}, \\
V_{12}=V_{21}=-P_{1}-P_{2}-P_{3}=-\beta_{1}\left(\varepsilon X_{1} X_{3}+X_{1} X_{6}\right)-\beta_{3} X_{1} X_{7}, \\
V_{22}=P_{1}+P_{2}+P_{3}+P_{4}+P_{5}+P_{6} \\
=\beta_{1}\left(\varepsilon X_{1} X_{3}+X_{1} X_{6}\right)+\beta_{3} X_{1} X_{7}+\gamma_{1}\left(\varepsilon X_{1} X_{3}+X_{1} X_{6}\right)+\eta_{1} X_{2}, \\
V_{23}=V_{32}=-P_{4}-P_{5}-P_{6}=-\gamma_{1}\left(\varepsilon X_{1} X_{3}+X_{1} X_{6}\right)-\eta_{1} X_{2}, \\
V_{33}=P_{4}+P_{5}+P_{6}+P_{7}+P_{8}=\gamma_{1}\left(\varepsilon X_{1} X_{3}+X_{1} X_{6}\right)+\eta_{1} X_{2}+v_{1} X_{3}+\delta_{1} X_{3}, \\
V_{37}=V_{73}=-P_{7}=-v_{1} X_{3}, \\
V_{44}=P_{10}+P_{11}+P_{9}=\beta_{2} X_{4} X_{6}+\beta_{4} X_{4} X_{7}+\beta_{2} \varepsilon X_{4} X_{7}, \\
V_{45}=V_{54}=-P_{10}-P_{11}-P_{9}=-\beta_{2} X_{4} X_{6}-\beta_{4} X_{4} X_{7}-\beta_{2} \varepsilon X_{4} X_{7}, \\
V_{55}=P_{10}+P_{11}+P_{13}+P_{12}+P_{14}=\beta_{2} X_{4} X_{6}+\beta_{4} X_{4} X_{7}+\gamma_{1} X_{5} X_{6}+\gamma_{1} \varepsilon X_{5} X_{3}+\eta_{2} X_{2}, \\
V_{56}=V_{65}=-P_{12}-P_{13}-P_{14}=-\gamma_{1} \varepsilon X_{5} X_{3}-\gamma_{1} X_{5} X_{6}-\eta_{2} X_{2}, \\
P_{13}+P_{14}+P_{15}+P_{16}=\gamma_{1} \varepsilon X_{5} X_{3}+\gamma_{1} X_{5} X_{6}+\eta_{2} X_{2}+\delta_{2} X_{3}+v_{2} X_{6}, \\
V_{67}=V_{76}=-P_{16}=-v_{2} X_{6}, \\
V_{77}=P_{16}+P_{17}+P_{18}+P_{7}=v_{2} X_{6}+\alpha X_{7}+\delta_{3} X_{3}+v_{1} X_{3}, \\
V_{78}=V_{87}=-P_{17}=\alpha X_{7}, V_{88}=P_{17}=\alpha X_{7} .
\end{gathered}
$$

The diffusion matrix $\Omega$ is symmetric and positive definite.
Using the approach of $[26,41,44]$, we construct a matrix $M$ such that $\Omega=M M^{T}$, where $M$ is an $8 \times 10$ matrix given as

$$
M=\left(\begin{array}{cccccccccc}
M_{1}^{1} & 0 & 0 & 0 & 0 & 0 & 0 & 0 & 0 & 0 \\
M_{2}^{1} & M_{2}^{2} & 0 & 0 & 0 & 0 & 0 & 0 & 0 & 0 \\
0 & M_{3}^{1} & M_{3}^{3} & M_{3}^{4} & 0 & 0 & 0 & 0 & 0 & 0 \\
0 & 0 & 0 & 0 & M_{4}^{5} & 0 & 0 & 0 & 0 & 0 \\
0 & 0 & 0 & 0 & M_{5}^{5} & M_{5}^{6} & 0 & 0 & 0 & 0 \\
0 & 0 & 0 & 0 & 0 & M_{6}^{6} & M_{6}^{7} & M_{6}^{8} & 0 & 0 \\
0 & 0 & M_{7}^{3} & 0 & 0 & 0 & M_{7}^{7} & 0 & M_{7}^{9} & M_{7}^{10} \\
0 & 0 & 0 & 0 & 0 & 0 & 0 & 0 & 0 & M_{8}^{10}
\end{array}\right),
$$

where

$$
\begin{gathered}
M_{1}^{1}=\sqrt{P_{1}+P_{2}+P_{3}}, \\
M_{2}^{1}=-\sqrt{P_{1}+P_{2}+P_{3}}, \\
M_{2}^{2}=\sqrt{P_{4}+P_{5}+P_{6}}, \\
M_{2}^{3}=-\sqrt{P_{4}+P_{5}+P_{6}}, \\
M_{3}^{3}=\sqrt{P_{7}}, \\
M_{3}^{4}=\sqrt{P_{8}}, \\
M_{4}^{5}=\sqrt{P_{9}+P_{10}+P_{11}}, \\
M_{5}^{5}=-\sqrt{P_{9}+P_{10}+P_{11}}, \\
M_{5}^{6}=\sqrt{P_{12}+P_{13}+P_{14}}, \\
M_{6}^{6}=-\sqrt{P_{12}+P_{13}+P_{14}}, \\
M_{6}^{7}=\sqrt{P_{16}}, \\
M_{6}^{7}=\sqrt{P_{15}}, \\
M_{7}^{3}=-\sqrt{P_{7}}, \\
M_{7}^{7}=-\sqrt{P_{16}}, \\
M_{7}^{9}=-\sqrt{P_{18}}, \\
M_{7}^{10}=\sqrt{P_{17}}, \\
M_{8}^{10}=-\sqrt{P_{17}} .
\end{gathered}
$$

Then, the Itô stochastic differential equation system has the form

$$
d(X(t))=f\left(X_{1}, X_{2}, X_{3}, X_{4}, X_{5}, X_{6}, X_{7}\right) d t+M \cdot d W(t),
$$

with initial condition

$$
X(0)=\left(X_{1}(0), X_{2}(0), X_{3}(0), X_{4}(0), X_{5}(0), X_{6}(0), X_{7}(0)\right)^{T},
$$




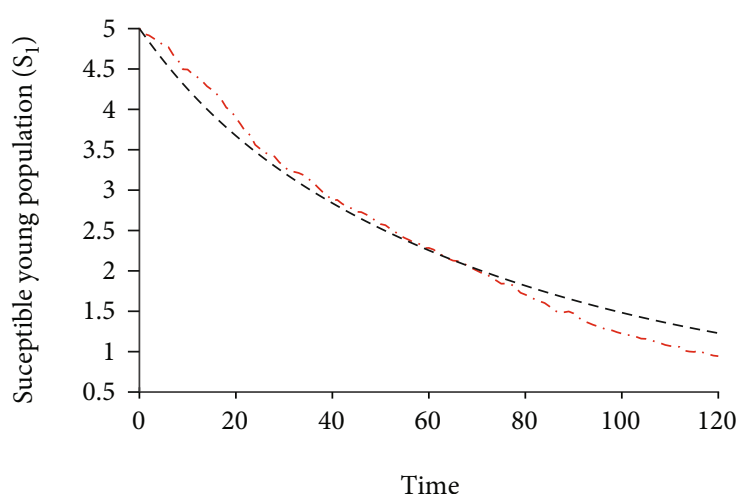

(a)

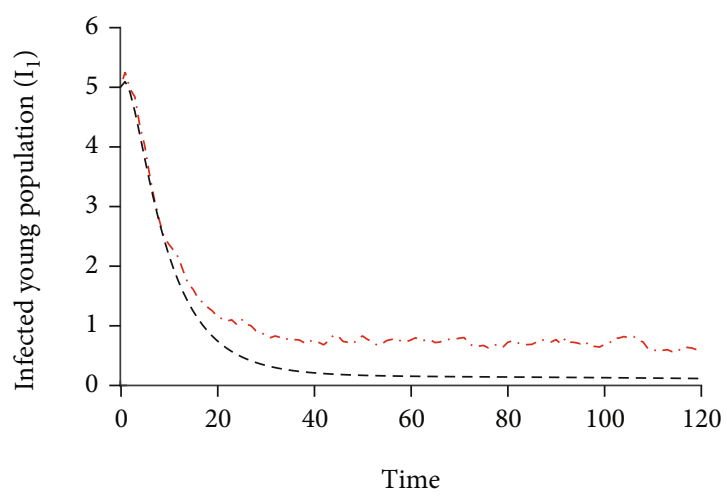

(c)

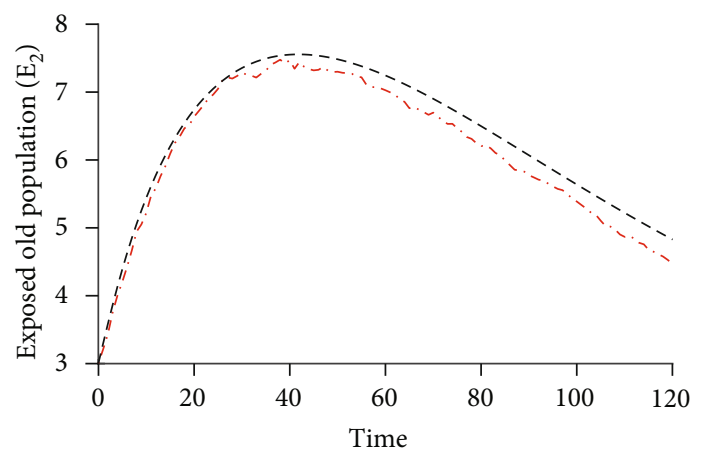

(e)

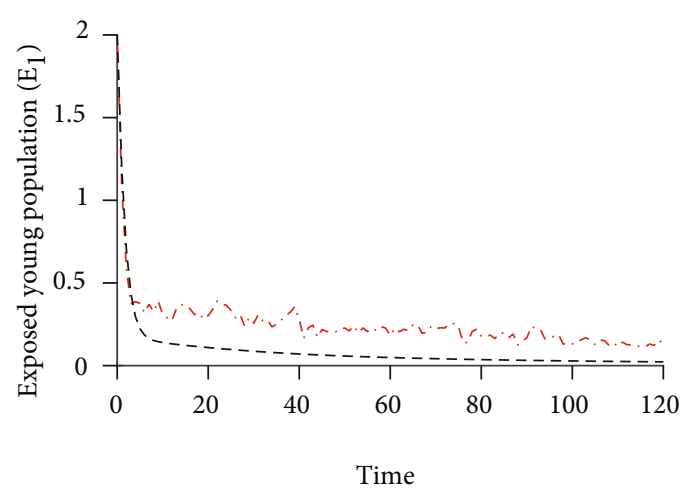

(b)

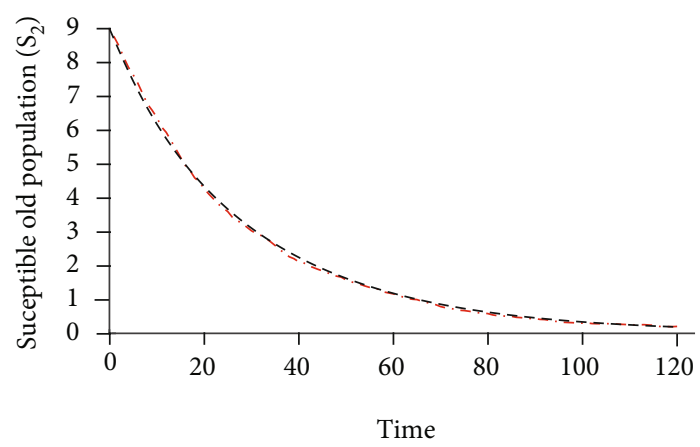

(d)

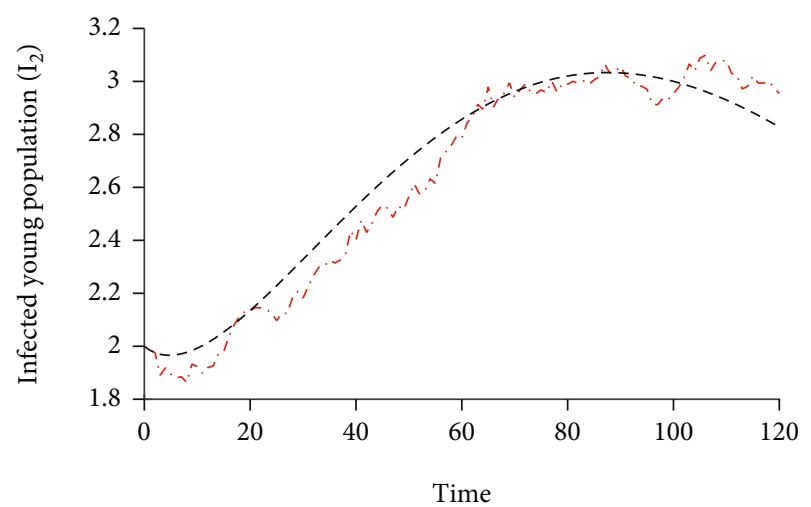

(f)

FIgURE 8: Continued. 


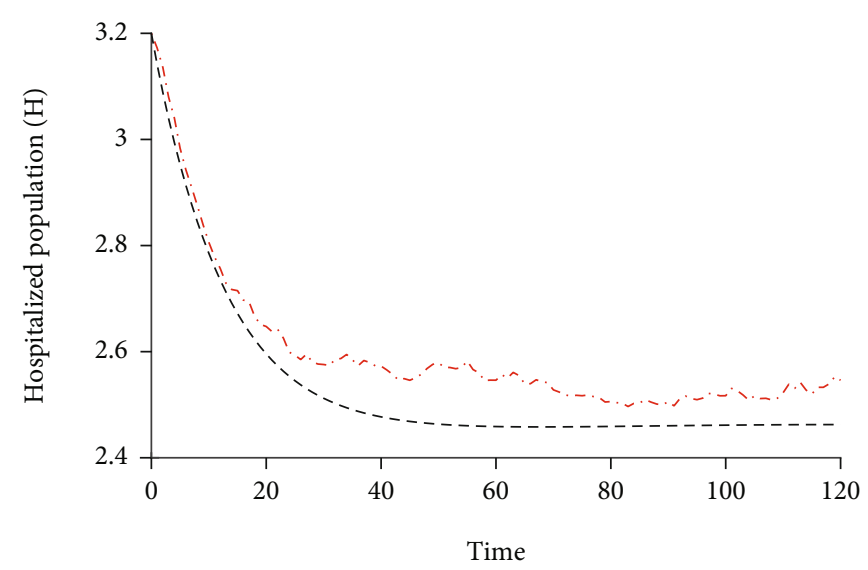

(g)

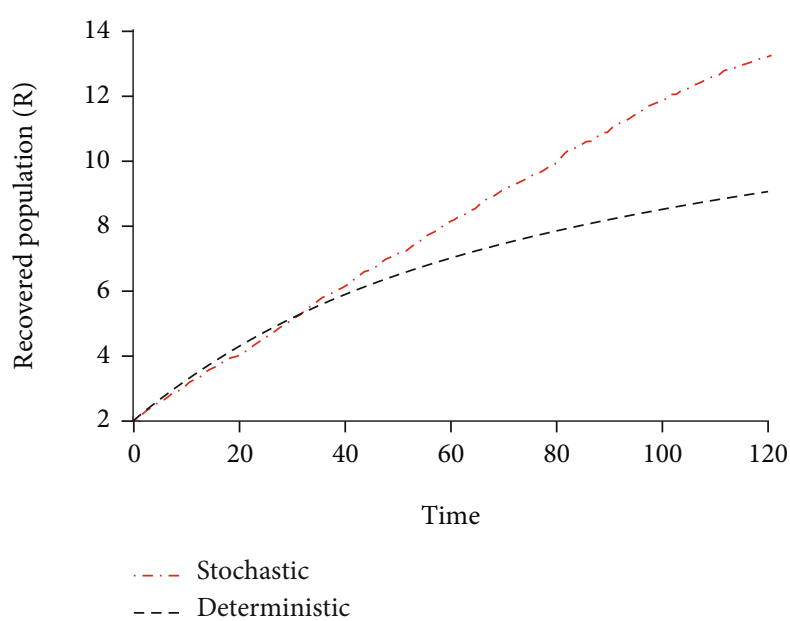

(h)

Figure 8: Time evolution of (a) $S_{1}$, (b) $E_{1}$, (c) $I_{1}$, (d) $S_{2}$, (e) $E_{2}$, (f) $I_{2}$, (g) $H$, and (h) $R$ for deterministic and stochastic systems (Italy).

and a Wiener process

$W(t)=\left(W_{1}(t), W_{2}(t), W_{3}(t), W_{4}(t), W_{5}(t), W_{6}(t), W_{7}(t), W_{8}(t), W_{9}(t), W_{10}(t)\right)^{T}$.

In view of the above facts, we construct the stochastic differential equation model as

$d S_{1}=\left(-\beta_{1} S_{1}\left(\varepsilon I_{1}+I_{2}\right)-\beta_{3} S_{1} H\right) d t+\sqrt{\beta_{1} S_{1}\left(\varepsilon I_{1}+I_{2}\right)+\beta_{3} S_{1} H} d W_{1}$,

$\begin{aligned} d E_{1}= & {\left[\beta_{1} S_{1}\left(\varepsilon I_{1}+I_{2}\right)+\beta_{3} S_{1} H-\gamma_{1} E_{1}\left(\varepsilon I_{1}+I_{2}\right)-\eta_{1} E_{1}\right] d t } \\ & -\sqrt{\beta_{1} S_{1}\left(\varepsilon I_{1}+I_{2}\right)+\beta_{3} S_{1} H} d W_{1}+\sqrt{\gamma_{1} E_{1}\left(\varepsilon I_{1}+I_{2}\right)+\eta_{1} E_{1}} d W_{2},\end{aligned}$

$$
\begin{aligned}
d I_{1}= & {\left[\eta_{1} E_{1}+\gamma_{1} E_{1}\left(\varepsilon I_{1}+I_{2}\right)-v_{1} I_{1}-\delta_{1} I_{1}\right] d t } \\
& -\sqrt{\gamma_{1} E_{1}\left(\varepsilon I_{1}+I_{2}\right)+\eta_{1} E_{1}} d W_{2}+\sqrt{v_{1} I_{1}} d W_{3}+\sqrt{\delta_{1} I_{1}} d W_{4},
\end{aligned}
$$

$d S_{2}=\left(-\beta_{2} S_{2}\left(\varepsilon I_{1}+I_{2}\right)-\beta_{4} S_{2} H\right) d t+\sqrt{\beta_{2} S_{2}\left(\varepsilon I_{1}+I_{2}\right)+\beta_{4} S_{2} H} d W_{5}$,

$$
\begin{aligned}
d E_{2}= & {\left[\beta_{2} S_{2}\left(\varepsilon I_{1}+I_{2}\right)+\beta_{4} S_{2} H-\gamma_{2} E_{2}\left(\varepsilon I_{1}+I_{2}\right)-\eta_{2} E_{2}\right] d t } \\
& -\sqrt{\beta_{2} S_{2}\left(\varepsilon I_{1}+I_{2}\right)+\beta_{4} S_{2} H} d W_{5}+\sqrt{\gamma_{2} E_{2}\left(\varepsilon I_{1}+I_{2}\right)+\eta_{2} E_{2}} d W_{6},
\end{aligned}
$$

$$
\begin{aligned}
d I_{2}= & {\left[\eta_{2} E_{2}+\gamma_{2} E_{2}\left(\varepsilon I_{1}+I_{2}\right)-v_{2} I_{2}-\delta_{2} I_{2}\right] d t } \\
& -\sqrt{\gamma_{2} E_{2}\left(\varepsilon I_{1}+I_{2}\right)+\eta_{2} E_{2}} d W_{6}+\sqrt{v_{2} I_{2}} d W_{7}+\sqrt{\delta_{2} I_{2}} d W_{8},
\end{aligned}
$$

$$
\begin{aligned}
d H= & {\left[v_{1} I_{1}+v_{2} I_{2}-\alpha H-\delta_{3} H\right] d t-\sqrt{v_{1} I_{1}} d W_{3} } \\
& -\sqrt{v_{2} I_{2}} d W_{7}+\sqrt{\alpha H} d W_{10}+\sqrt{\delta_{3} H} d W_{9}
\end{aligned}
$$

$$
d R=[\alpha H] d t-\sqrt{\alpha H} d W_{10}
$$

\section{Stochastic Simulation Results}

To emphasize the impact of stochasticity in the system, we simulate the stochastic model shown in system (28) by using Euler-Maruyama method [45]. For this purpose, we use the following set of parameter values given in Tables 2 and 3 . We perform simulations of system (28) for 120 days and 100 simulation runs. First, we compare the mean of 100 runs of stochastic model simulation with the results of corresponding deterministic model and plot the time series of all the variables; see Figure 8 for Italy and Figure 9 for Spain. From these figures, we observe that the mean of 100 runs of stochastic simulation is very close to the simulation results of the deterministic model for the susceptible population $\left(S_{1}\right)$ and $\left(S_{2}\right)$, whereas for the other populations a small deviation between stochastic and deterministic simulations results is observed.

In order to understand these results better, we also plot the distributions of the infected young $\left(I_{1}\right)$ and old $\left(I_{2}\right)$ populations at the 50th, 80th, and 120th days. These can be seen in Figures 10 and 11 for Italy and Spain, respectively. One can easily see the change in distribution of the population as time progresses.

\section{Results and Discussion}

With an unprecedented global health burden, the COVID19 pandemic has changed the behavior of societies around the globe which were significantly affected by the extreme measures implemented to control disease transmission.

In this paper, a mathematical model for COVID-19 transmission is formulated and analyzed. We computed the disease-free equilibrium and the basic reproduction number $R_{0}$. Sensitivity analysis was performed to find the key parameters that are most sensitive to basic reproduction number $R_{0}$. The deterministic model was extended to its 


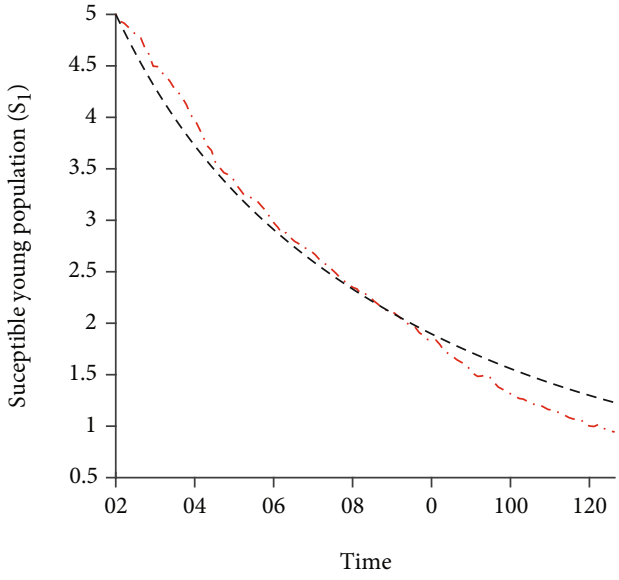

(a)

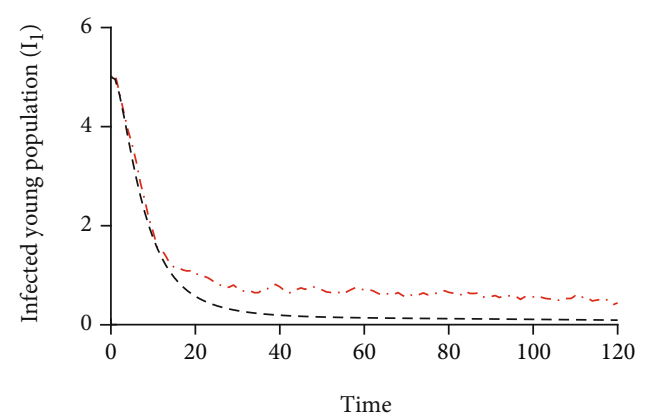

(c)

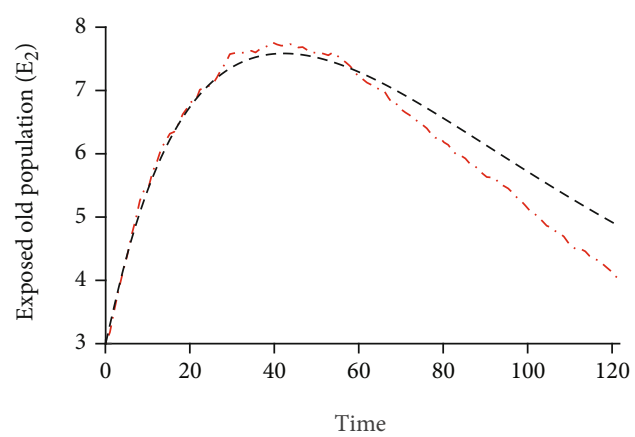

(e)

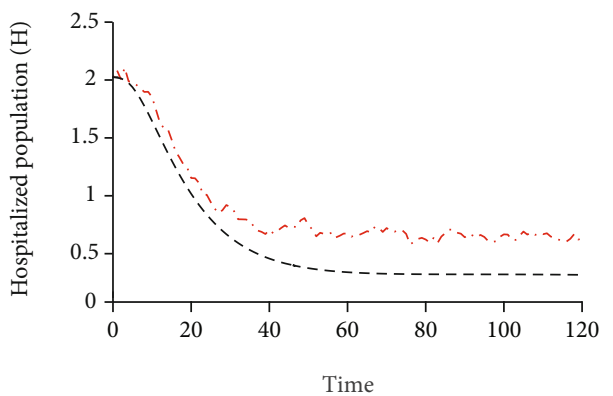

(g)

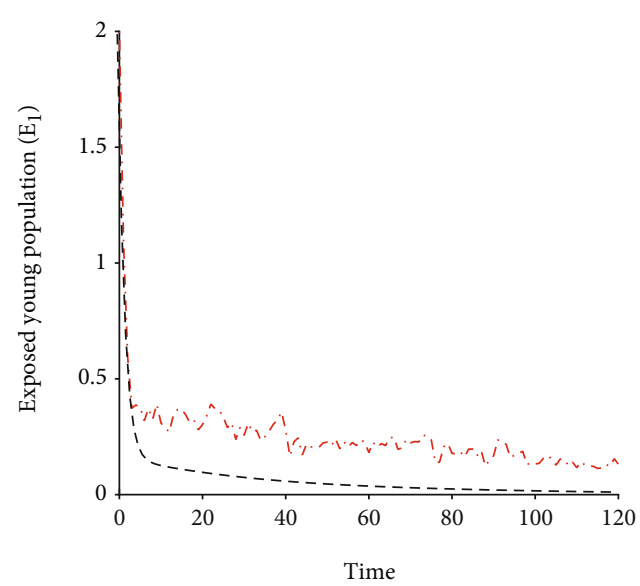

(b)

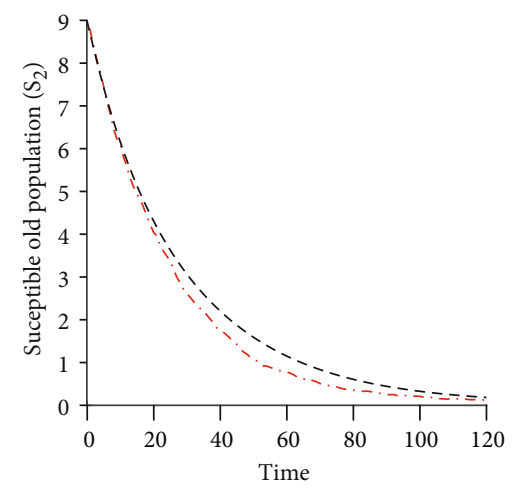

(d)

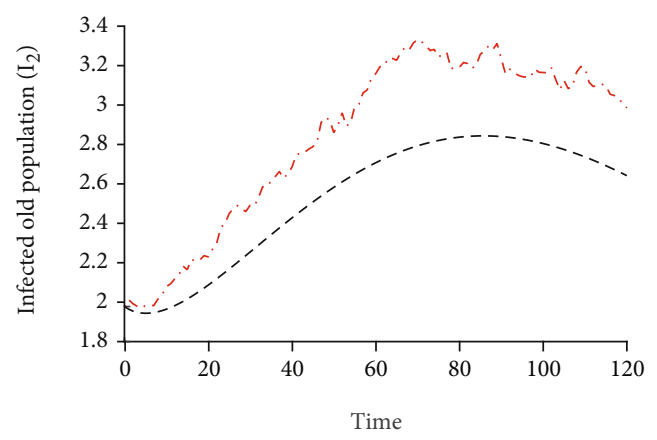

(f)

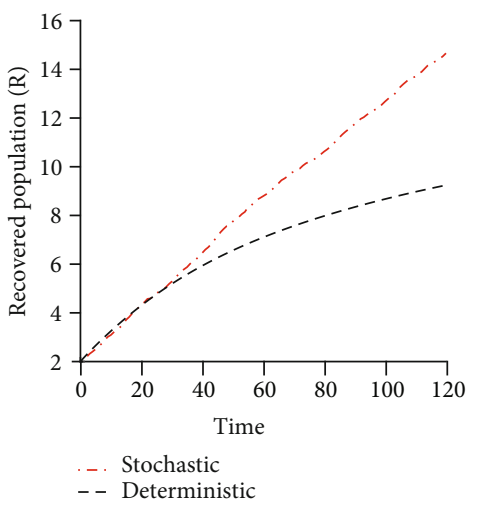

(h)

Figure 9: Time evolution of (a) $S_{1}$, (b) $E_{1}$, (c) $I_{1}$, (d) $S_{2}$, (e) $E_{2}$, (f) $I_{2}$, (g) $H$, and (h) $R$ for deterministic and stochastic systems (Spain). 


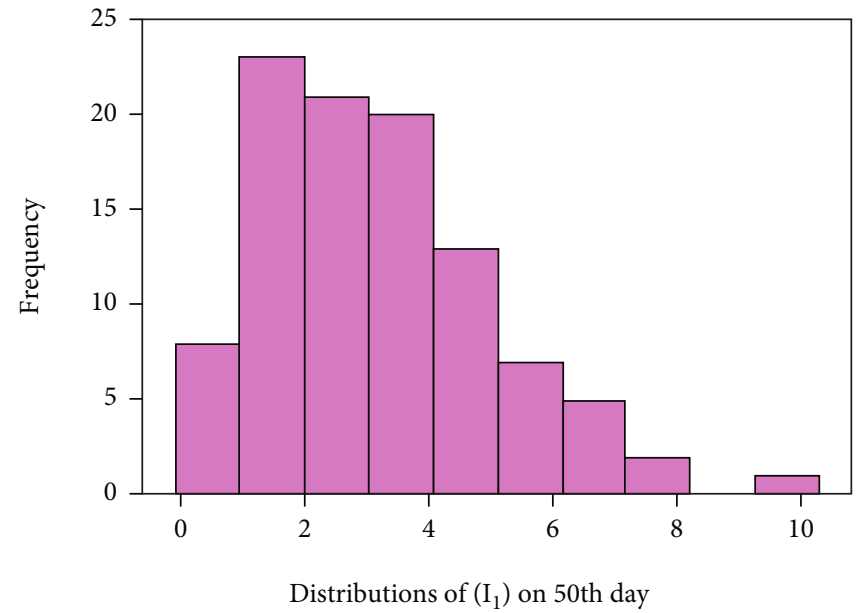

(a)

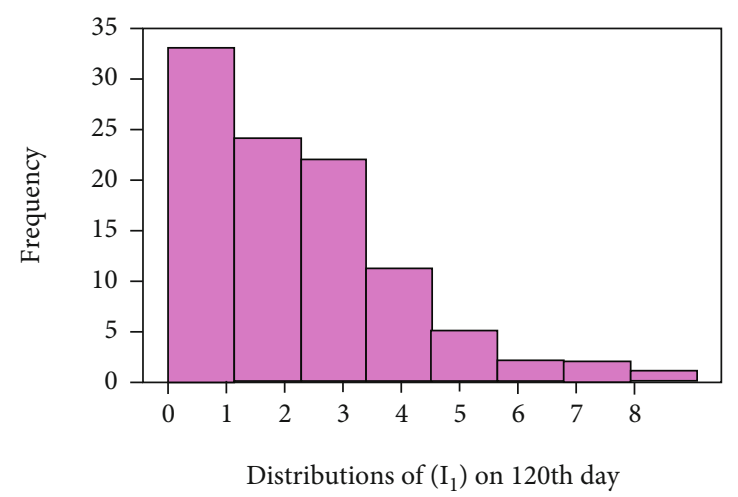

(c)

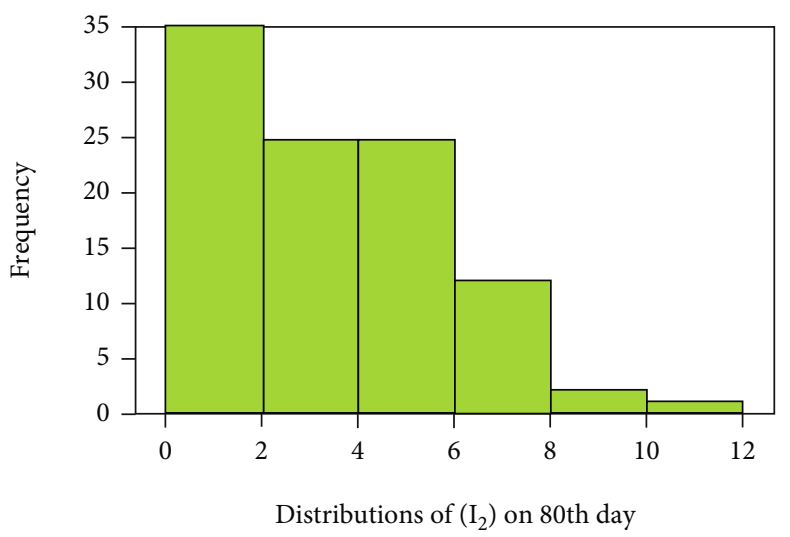

(e)

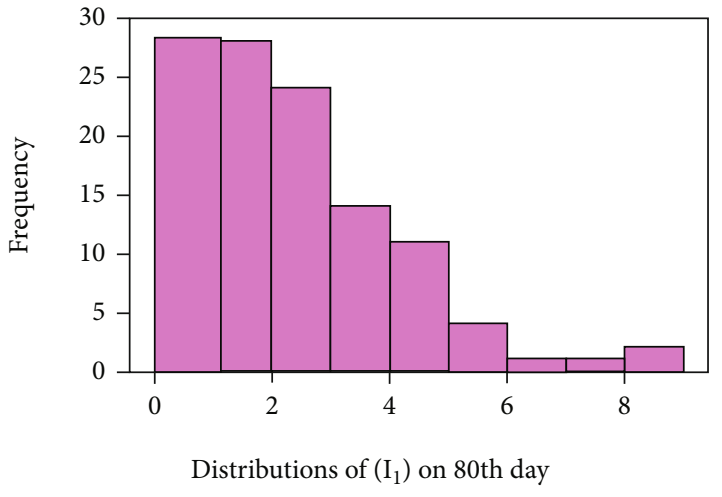

(b)

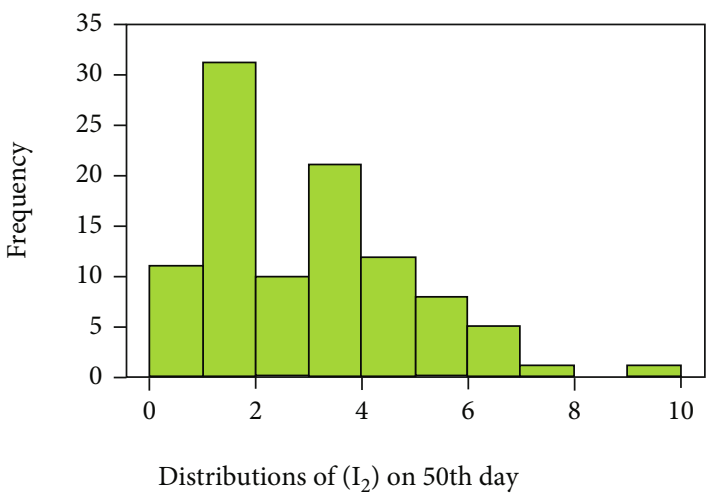

(d)

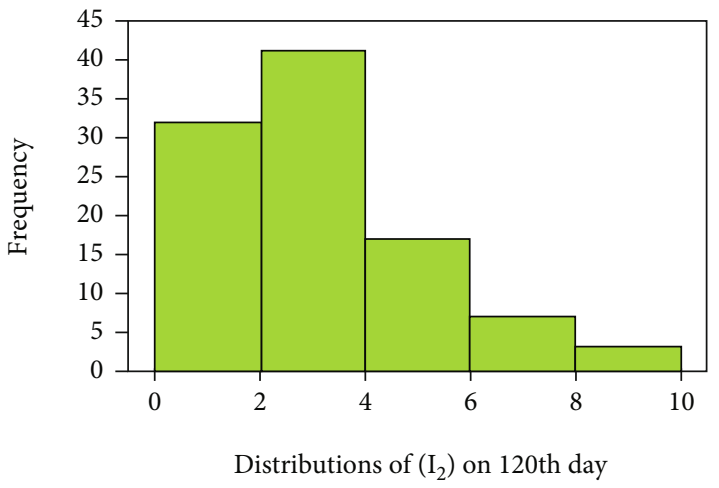

(f)

Figure 10: Distributions of symptomatic infected population $I_{1}$ and $I_{2}$ on days 50, 80, and 120 (Italy).

stochastic counterpart. Using numerical simulations, the distributions of young infected and old infected populations have shown the behaviors of trajectories when the model is affected by stochastic perturbations.

In this work, we have introduced a new parameter $\varepsilon$ that plays a very important role to reduce the infectivity of the young population. While $\varepsilon>1$ represents higher infectivity of young population than the infectivity of older population, the assumption of $\varepsilon<1$ represents a lower infectivity of young population than the infectivity of older population. A detailed analysis of this specific parameter is ongoing.

Results presented here support the reduction of the transmission rate between human to human, by the proper use of nonpharmaceutical intervention and vaccination, to affect the most the dynamics of the pandemic. Moreover, a fast detection of the infected individuals would lead to a better treatment, decreasing the disease mortality rate in the population. We would like to emphasize that this is an 


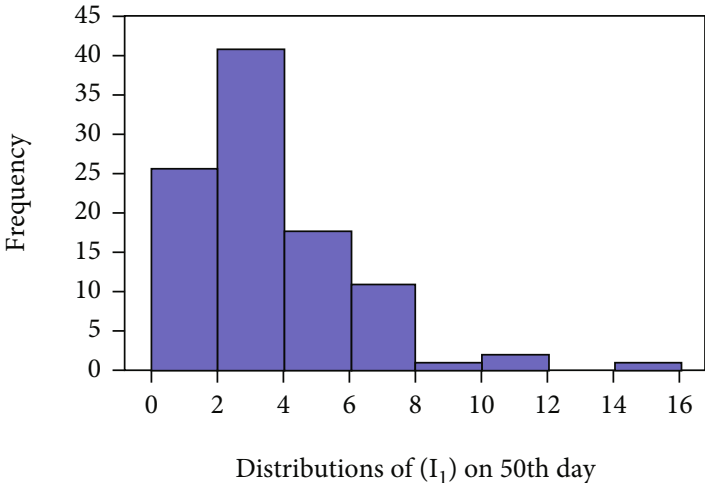

(a)

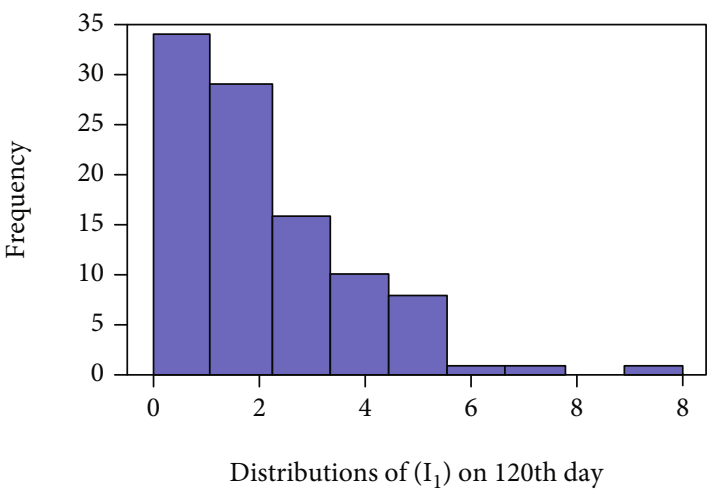

(c)

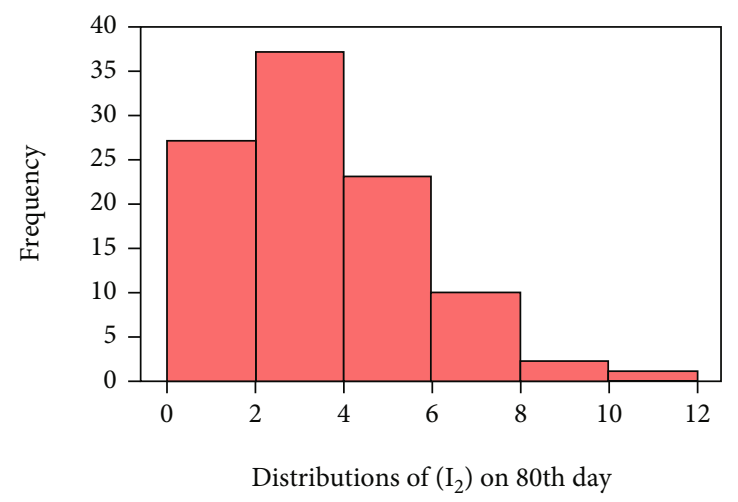

(e)

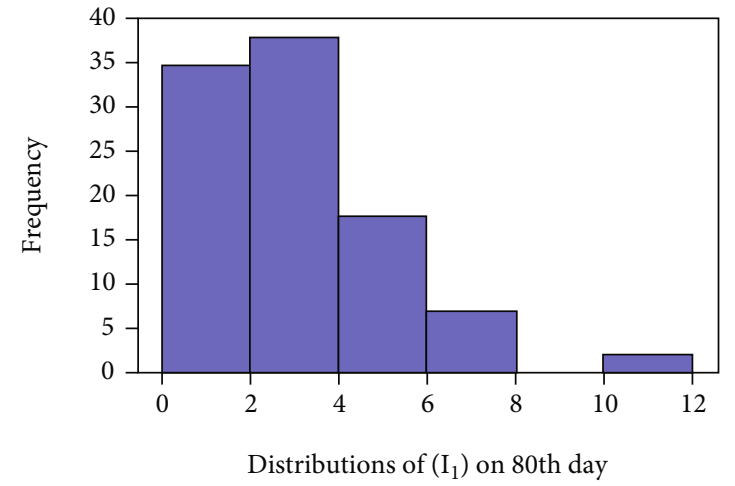

(b)

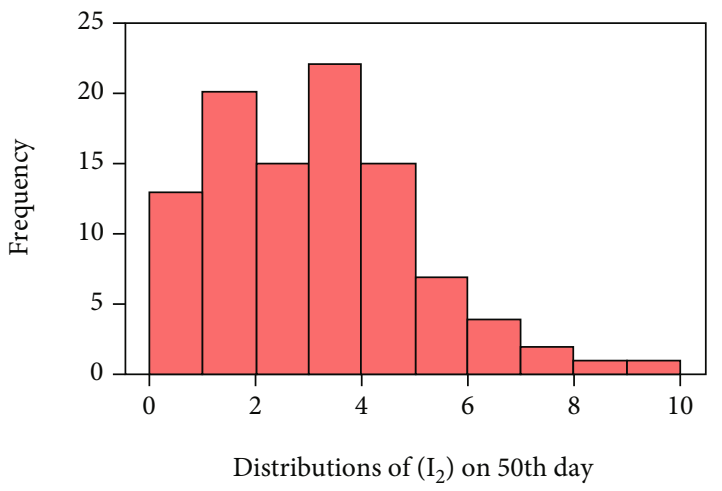

(d)

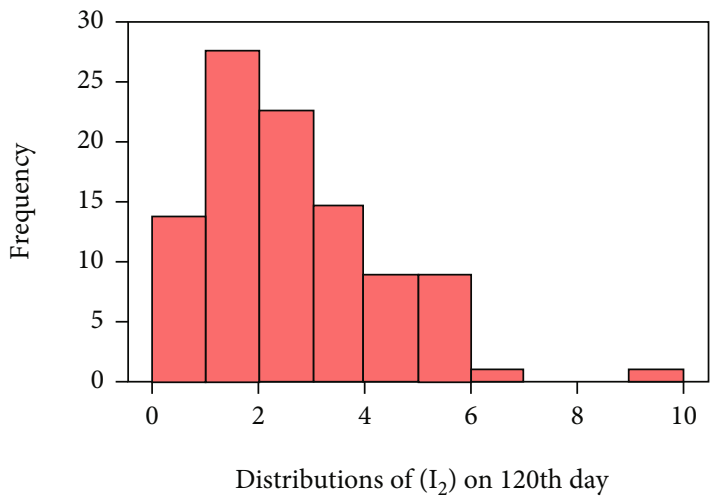

(f)

FIgUre 11: Distributions of symptomatic infected population $I_{1}$ and $I_{2}$ on days 50, 80, and 120 (Spain).

ongoing work and models will be refined and extended, using the present model as a baseline for future research. As an example, applied to the Basque Country, Spain, scenario, the incorporation of asymptomatic classes for both young and old groups is proposed to evaluate its impact on the control measures implemented over the pandemic time.

\section{Abbreviations}

ANA: Antinuclear antibodies

APC: Antigen-presenting cells

IRF: Interferon regulatory factor.

\section{Data Availability}

Previously reported data on COVID-19 cases for Italy and Spain were used to support this study and are available at doi:10.1002/mma.7344. These prior studies (and datasets) are cited at relevant places within the text as references $[20,21]$.

\section{Conflicts of Interest}

The authors declare no potential conflict of interests. 


\section{Authors' Contributions}

M.A. supervised the development of this study. A.K.S. conceived and performed the numerical simulations. All authors contributed to the development of the modeling framework, analysis of the results, and the writing of the manuscript.

\section{Acknowledgments}

This research is supported by the Basque Government through the "Mathematical Modeling Applied to Health" Project, BERC 2018-2021 program, and by the Spanish Ministry of Sciences, Innovation and Universities: BCAM Severo Ochoa accreditation SEV-2017-0718.

\section{References}

[1] World Health Organization, "Coronavirus disease (COVID19)," June 2021, https://www.who.int/health-topics/ coronavirustab $=$ tab 1 .

[2] M. Aguiar and N. Stollenwerk, "Condition-specific mortality risk can explain differences in COVID-19 case fatality ratios around the globe," Public Health, vol. 188, pp. 18-20, 2020.

[3] M. Voysey, S. A. C. Clemens, S. A. Madhi et al., "Safety and efficacy of the ChAdOx1 nCoV-19 vaccine (AZD1222) against SARS- CoV-2: an interim analysis of four randomised controlled trials in Brazil, South Africa, and the UK," The Lancet, vol. 397, no. 10269, pp. 99-111, 2021.

[4] F. P. Polack, S. J. Thomas, N. Kitchin et al., "Safety and efficacy of the BNT162b2 mRNA Covid-19 vaccine," New England Journal of Medicine, vol. 383, no. 27, pp. 2603-2615, 2020.

[5] L. R. Baden, H. M. el Sahly, B. Essink et al., "Efficacy and safety of the mRNA-1273 SARS-CoV-2 vaccine," New England Journal of Medicine, vol. 384, no. 5, pp. 403-416, 2021.

[6] J. Sadoff, G. Gray, A. Vandebosch et al., "Safety and efficacy of single-dose Ad26.COV2.S vaccine against Covid-19," New England Journal of Medicine, vol. 384, no. 23, pp. 2187-2201, 2021.

[7] N. Dagan, N. Barda, E. Kepten et al., "BNT162b2 mRNA Covid-19 vaccine in a nationwide mass vaccination setting," New England Journal of Medicine, vol. 384, no. 15, pp. 14121423, 2021.

[8] V. J. Hall, S. Foulkes, A. Saei et al., "COVID-19 vaccine coverage in health-care workers in England and effectiveness of BNT162b2 mRNA vaccine against infection (SIREN): a prospective, multicentre, cohort study," The Lancet, vol. 397, no. 10286, pp. 1725-1735, 2021.

[9] M. A. Luís Mateus and N. Stollenwerk, "Bayesian estimation of vaccine efficacy," in Proceedings of the 15th International Conference on Mathematical Methods in Science and Engineering, pp. 794-802, Cadiz, Spain, 2015.

[10] M. Aguiar, N. Stollenwerk, and S. B. Halstead, "The impact of the newly licensed dengue vaccine in endemic countries," PLOS Neglected Tropical Diseases, vol. 10, no. 12, article e0005179, 2016.

[11] M. Aguiar, N. Stollenwerk, and S. B. Halstead, "The risks behind Dengvaxia recommendation," The Lancet Infectious Diseases, vol. 16, no. 8, pp. 882-883, 2016.

[12] M. Aguiar, S. B. Halstead, and N. Stollenwerk, "Consider stopping Dengvaxia administration without immunological screening," Expert Review of Vaccines, vol. 16, no. 4, pp. 301302, 2016.

[13] M. Aguiar and N. Stollenwerk, "Dengvaxia efficacy dependency on serostatus: a closer look at more recent data," Clinical Infectious Diseases, vol. 66, no. 4, pp. 641-642, 2017.

[14] M. Aguiar and N. Stollenwerk, "Dengvaxia: age as surrogate for serostatus," The Lancet Infectious Diseases, vol. 18, no. 3, p. 245, 2018.

[15] M. Aguiar, "Dengue vaccination: a more ethical approach is needed," The Lancet, vol. 391, no. 10132, pp. 1769-1770, 2018.

[16] S. B. Halstead, L. C. Katzelnick, P. K. Russell et al., "Ethics of a partially effective dengue vaccine: lessons from the Philippines," Vaccine, vol. 38, no. 35, pp. 5572-5576, 2020.

[17] M. Aguiar and N. Stollenwerk, "The impact of serotype crossprotection on vaccine trials: DENVax as a case study," Vaccine, vol. 8, no. 4, p. 12, 2020.

[18] N. Stollenwerk, J. Mar, J. Bidaurrazaga Van-Dierdonck, O. Ibarrondo, C. Estadilla, and M. Aguiar, Modeling COVID19 Vaccine Efficacy and Coverage towards Herd-Immunity in the Basque Country, medRxiv, Spain, 2021.

[19] M. Aguiar, J. B. Van-Dierdonck, J. Mar, and N. Stollenwerk, "The role of mild and asymptomatic infections on COVID19 vaccines performance: a modeling study," Journal of Advanced Research, 2021.

[20] November 2021, https://www.worldometers.info/coronavirus/ country/spain/.

[21] November 2021, https://www.worldometers.info/coronavirus/ country/italy/.

[22] “The statistics portal, Statista," February 2018, https://www .statista.com/statistics/1105596/covid-19-mortality-rate-byage-group-in-spain-march/.

[23] “The statistics portal, Statista," February 2018, https://www .statista.com/statistics/1105061/coronavirus-deaths-byregion-in-italy/.

[24] M. Aguiar and N. Stollenwerk, "SHAR and effective SIR models: from dengue fever toy models to a COVID-19 fully parametrized SHARUCD framework," Communication in Biomathematical Sciences, vol. 3, no. 1, pp. 60-89, 2020.

[25] M. Aguiar, G. Dosi, D. Knopoff, and M. E. Virgillito, "A multiscale network-based model of contagion dynamics: heterogeneity, spatial distancing and vaccination," Mathematical Models and Methods in Applied Sciences, pp. 1-30, 2021.

[26] M. Aguiar, E. Millán Ortuondo, J. Bidaurrazaga Van-Dierdonck, J. Mar, and N. Stollenwek, "Modelling COVID 19 in the Basque Country from introduction to control measure response," Scientific Reports, vol. 10, p. 17306, 2020.

[27] M. Aguiar, J. Bidaurrazaga Van-Dierdonck, J. Mar et al., "Critical fluctuations in epidemic models explain COVID-19 postlockdown dynamics," Scientific Reports, vol. 11, p. 13839, 2021.

[28] M. Aguiar, J. Bidaurrazaga Van-Dierdonck, and N. Stollenwerk, "Reproduction ratio and growth rates: measures for an unfolding pandemic," PLoS One, vol. 15, article e0236620, 2020.

[29] “The epidemiological SHARUCD model dashboard," July 2021, https://wp.bcamath.org/news/en/epidemiologicalsharucd-model/introduction.

[30] J. Chu, "A statistical analysis of the novel coronavirus (COVID-19) in Italy and Spain," PLoS One, vol. 16, no. 3, article e0249037, 2021.

[31] A. K. Srivastav, P. K. Tiwari, P. K. Srivastava, M. Ghosh, and Y. Kang, "A mathematical model for the impacts of face mask, 
hospitalization and quarantine on the dynamics of COVID-19 in India: deterministic vs. stochastic," Mathematical Biosciences and Engineering, vol. 18, no. 1, pp. 182-213, 2021.

[32] D. Olabode, J. Culp, A. Fisher, A. Tower, D. Hull-Nye, and $\mathrm{X}$. Wang, "Deterministic and stochastic models for the epidemic dynamics of COVID-19 in Wuhan," China. Mathematical Biosciences and Engineering, vol. 18, no. 1, pp. 950-967, 2021.

[33] A. K. Srivastav, M. Ghosh, X.-Z. Li, and L. Cai, "Modeling and optimal control analysis of COVID-19: case studies from Italy and Spain," Mathematical Methods in the Applied Sciences, vol. 44, no. 11, pp. 9210-9223, 2021.

[34] "Coronavirus disease (COVID-19)," https://www.who.int/ news-room/q-a-detail/q-a-coronaviruses.

[35] N. M. Ferguson, D. Laydon, G. Nedjati-Gilani et al., Impact of non-pharmaceutical interventions (NPIs) to reduce COVID19 mortality and healthcare demand, Imperial College London, 2020.

[36] B. Tang, N. L. Bragazzi, Q. Li, S. Tang, Y. Xiao, and J. Wu, "An updated estimation of the risk of transmission of the novel coronavirus (2019-nCov)," Infectious Disease Modelling, vol. 5, pp. 248-255, 2020.

[37] F. Zhou, T. Yu, R. du et al., "Clinical course and risk factors for mortality of adult inpatients with COVID-19 in Wuhan, China: a retrospective cohort study," The Lancet, vol. 395, no. 10229, pp. 1054-1062, 2020.

[38] P. V. Driessche and J. Watmough, "Reproduction numbers and subthreshold endemic equilibria for compartmental models of disease transmission," Mathematical Biosciences, vol. 180, no. 1, pp. 29-48, 2008.

[39] N. Chitnis, J. M. Hyman, and J. M. Cushing, "Determining important pa- rameters in the spread of malaria through the sensitivity analysis of a mathematical model," Bulletin of Mathematical Biology, vol. 70, no. 5, pp. 1272-1296, 2008.

[40] N. Stollenwerk and V. Jansen, Population Biology and Criticality: From Critical Birth-Death Processes to Self-Organized Criticality in Mutation Pathogen Systems, World Scientific, London, 2011.

[41] E. J. Allen, L. J. S. Allen, A. Arciniega, and P. Greenwood, "Construction of equivalent stochastic differential equation models," Stochastic Analysis and Application, vol. 26, pp. 274-297, 2008.

[42] N. G. van Kampen, Stochastic Processes in Physics and Chemistry, North-Holland, Amsterdam, 1992.

[43] C. W. Gardiner, Handbook of Stochastic Methods, Springer, New York, 1985.

[44] Y. Yuan and L. J. S. Allen, "Stochastic models for virus and immune system dynamics," Mathematical Biosciences, vol. 234, no. 2, pp. 84-94, 2011.

[45] P. E. Kloeden and E. Platen, Numerical Solution of Stochastic Differential Equations, Springer, Berlin, 1992. 